\title{
Effect of robotic-assisted gait training on objective biomechanical measures of gait in persons post-stroke: a systematic review and meta-analysis
}

\author{
Heidi Nedergård ${ }^{1 *}$, Ashokan Arumugam² ${ }^{2}$ Marlene Sandlund ${ }^{1}$, Anna Bråndal ${ }^{1}$ and Charlotte K. Häger ${ }^{1}$
}

\begin{abstract}
Background: Robotic-Assisted Gait Training (RAGT) may enable high-intensive and task-specific gait training poststroke. The effect of RAGT on gait movement patterns has however not been comprehensively reviewed. The purpose of this review was to summarize the evidence for potentially superior effects of RAGT on biomechanical measures of gait post-stroke when compared with non-robotic gait training alone.

Methods: Nine databases were searched using database-specific search terms from their inception until January 2021. We included randomized controlled trials investigating the effects of RAGT (e.g., using exoskeletons or endeffectors) on spatiotemporal, kinematic and kinetic parameters among adults suffering from any stage of stroke. Screening, data extraction and judgement of risk of bias (using the Cochrane Risk of bias 2 tool) were performed by 2-3 independent reviewers. The Grading of Recommendations Assessment Development and Evaluation (GRADE) criteria were used to evaluate the certainty of evidence for the biomechanical gait measures of interest.

Results: Thirteen studies including a total of 412 individuals (mean age: 52-69 years; 264 males) met eligibility criteria and were included. RAGT was employed either as monotherapy or in combination with other therapies in a subacute or chronic phase post-stroke. The included studies showed a high risk of bias $(n=6)$, some concerns $(n=6)$ or a low risk of bias $(n=1)$. Meta-analyses using a random-effects model for gait speed, cadence, step length (nonaffected side) and spatial asymmetry revealed no significant differences between the RAGT and comparator groups, while stride length (mean difference [MD] $2.86 \mathrm{~cm}$ ), step length (affected side; MD $2.67 \mathrm{~cm}$ ) and temporal asymmetry calculated in ratio-values (MD 0.09) improved slightly more in the RAGT groups. There were serious weaknesses with almost all GRADE domains (risk of bias, consistency, directness, or precision of the findings) for the included outcome measures (spatiotemporal and kinematic gait parameters). Kinetic parameters were not reported at all.

Conclusion: There were few relevant studies and the review synthesis revealed a very low certainty in current evidence for employing RAGT to improve gait biomechanics post-stroke. Further high-quality, robust clinical trials on RAGT that complement clinical data with biomechanical data are thus warranted to disentangle the potential effects of such interventions on gait biomechanics post-stroke.
\end{abstract}

Keywords: Cerebrovascular accident, Powered exoskeleton, Walk, Rehabilitation, Literature synthesis

*Correspondence: heidi.nedergard@umu.se

1 Department of Community Medicine and Rehabilitation, Physiotherapy, Umeå University, Umeå, Sweden

Full list of author information is available at the end of the article original author(s) and the source, provide a link to the Creative Commons licence, and indicate if changes were made. The images or other third party material in this article are included in the article's Creative Commons licence, unless indicated otherwise in a credit line to the material. If material is not included in the article's Creative Commons licence and your intended use is not permitted by statutory regulation or exceeds the permitted use, you will need to obtain permission directly from the copyright holder. To view a copy of this licence, visit http://creativecommons.org/licenses/by/4.0/. The Creative Commons Public Domain Dedication waiver (http://creativeco mmons.org/publicdomain/zero/1.0/) applies to the data made available in this article, unless otherwise stated in a credit line to the data. 


\section{Background}

Technology-assisted interventions to enhance gait rehabilitation post-stroke are highly interesting from a clinical perspective. Robotic-assisted gait training (RAGT) employs electromechanical devices that assist stepping cycles by supporting body weight while automatizing the gait process through support and facilitation of movement in one or several lower limb joints. RAGT is suggested to be less energy-consuming and cardiorespiratory demanding when compared with walking without a robot [1]. Implementing RAGT may thus enable higher intensities and longer, task-specific training sessions when compared with non-robotic gait training.

Various forms of robotic devices are commercially available and they are commonly categorized according to the support they apply [2]. Treadmill-based RAGT ( $t$-RAGT) is most commonly used in combination with body weight support [3]. This is either performed with end-effector robots that drive two footplates, simulating the phases of the gait, or with exoskeleton orthoses that move the lower body extremity joints in coordination with the phases of gait. Overground RAGT (o-RAGT) is provided by wearable powered exoskeletons that allow a person to walk overground on hard and flat surfaces [4], supposedly enabling the user to experience increased proprioceptive input when compared with the stationary treadmill training [5].

Earlier reviews revealed that RAGT, together with conventional physiotherapy, might have a slightly better or similar positive effect on gait speed and ambulation when compared with conventional gait training alone [6-16]. However, the need for a broadened perspective in the evaluation of gait ability after RAGT post-stroke has been highlighted $[13,15,17,18]$. The International Classification of Functioning, Disability and Health (ICF), advocated by the World Health Organization, is a classification system widely used in clinical practice [19]. It is a foundation for understanding the patient's personal and environmental resources and limitations, hence also used when evaluating rehabilitation effects from different perspectives. The classification system identifies three domains of a health condition: (1) body function (physiological and psychological) and structure (related to organs, limbs, etc.), (2) activity (related to the execution of a task, and (3) participation (related to involvement in a real-life situation). Although the domains are interrelated, measurements of all domains and contextual factors are necessary to describe a person's condition from a holistic point of view. In a 2013 review, Geroin and colleagues [20] emphasize that a comprehensive post-intervention evaluation of RAGT, such as that of any other intervention, should use outcome measures that include all domains of the ICF. In general, tests that evaluate walking ability post-stroke address activity limitations alone (6 min Walk Test, Timed Up and Go, Functional Ambulation Category). These tests might fail to identify restrictions related to the domain of body function and structure since they do not investigate specific gait characteristics, such as coordination, muscle power, joint mobility or extremity positions during gait. In persons post-stroke, gains in walking ability following rehabilitation may be considered a result of the restitution of underlying impairments. However, improvements in activity measures could also partly be explained by an adaptation of non-optimal movement strategies that compensate for existing deficits [21, 22]. A paradigm shift has occurred in the research area of gait rehabilitation post-stroke [23], claiming that rehabilitation methods that stimulate the nervous system's ability to recover a normalized movement pattern should be preferred before those encouraging compensation for impaired mobility, motor control, and balance. In line with this, the quantitative evaluation of gait quality and movement pattern may allow for differentiation of recovery mechanisms and foster a deeper understanding of the effects of different gait rehabilitation interventions post-stroke [18, $23,24]$. To manage this, various biomechanical variables of temporal (related to time) or spatial (related to distance) information have been applied. These are derived from kinematic (parameters of registered position, motion and/or marker trajectories of interest to describe the locomotion pattern) or kinetic (registered forces that act on the body during movement) measures of gait [24]. A gait-assisting robot aims to replicate a movement pattern that is as close to normal as possible with regards to temporal and spatial parameters. It is also believed to generate more repetitions with regards to the number of steps during one training session as compared with non-robotic gait training. RAGT could thus be assumed to improve gait quality to a greater extent than training without a robot by normalizing the movement pattern and increasing training volume with a carryover effect to when the person is walking without the assisting robot. This review aims to summarize the level of evidence for any potential superior effects of RAGT (with or without a combination of non-robotic training) compared with non-robotic training alone on post-stroke gait movement pattern quantified with objective biomechanical measures.

\section{Methods \\ Design and registration}

This review followed a protocol pre-registered in PROSPERO (CRD42020168846). The Preferred Reporting Items for Systematic review and Meta-Analysis (PRISMA) statement was used as a framework to 
document the objectives, methods and findings of the review $[25,26]$. Following PRISMA recommendations, the research question and the eligibility criteria were framed using the PICO approach (representing the patient population $(\mathrm{P})$, the interventions $(\mathrm{I})$, the comparator group $(\mathrm{C})$, and the outcome $(\mathrm{O})$, and the study design chosen [27].

\section{Eligibility criteria Type of studies}

This review included only randomized clinical trials (RCT) that investigated the effects of robotic-assisted gait training using instrumented gait analysis to evaluate gait performance during overground or treadmill walking. All other study designs were excluded.

\section{Type of participants}

This review included adult participants $(\geq 18$ years of age) in an acute, subacute or chronic phase post-stroke. The stroke could be due to haemorrhagic or ischemic causes. No restrictions were made regarding the functional ability or gender of the participants with regards to inclusion.

\section{Type of intervention and comparator groups}

RAGT for gait rehabilitation in either an inpatient or outpatient setting was mandatory for inclusion in this review. RAGT was defined as robotic-assisted gait training using an electromechanical device to assist the stepping cycles during walking. The devices could be either end-effectors or exoskeletons for treadmill gait training or exoskeletons used for overground gait training [3]. Contemporary evidence and recommendations suggest that RAGT should complement, not replace, existing gait rehabilitation and non-robotic physical therapies [3, 13]. We therefore also included studies using a combination of RAGT and other therapies such as conventional physiotherapy training or functional electrical stimulation (FES). All studies were nevertheless required to have at least one comparator group performing active, nonrobotic gait rehabilitation post-stroke. Non-weight-bearing interventions that used non-interactive devices for delivering continuous passive motion (e.g., an isokinetic apparatus for passive knee flexion [28]), or devices used for seated or standing lower extremity training (e.g., the MotionMaker $^{\text {TM }}$ [29], the Rutgers Ankle [30] or the Active Knee Rehabilitation Orthotic Devices (AKROD) [31]) were excluded.

\section{Outcomes of interest}

Instrumented gait analysis was to be performed in either a laboratory or a clinical setting using devices that register kinematic or kinetic parameters during walking: a 3-dimensional (3D) or 2-dimensional (2D) motion capture system, an optoelectrical or inertial system, a gait or pressure mat, force shoes, a magnetic or acoustic tracking system, etc. Outcome measures of interest were parameters related to temporal and spatial information based on kinematics and kinetics. Studies that assessed gait biomechanics during RAGT, while wearing the robotic device, or immediately after only a single training session were excluded. Results of biomechanical outcomes measured solely by clinical testing, such as gait speed evaluated with a stopwatch or cadence reported from observations were also excluded.

\section{Search strategy}

One reviewer $(\mathrm{HN})$ performed a systematic search in the following databases: PubMed, Web of Science, EBSCO (Cumulative Index to Nursing and Allied Health Literature [CINAHL], Allied and Complementary Medicine [AMED], Academic Search Premier, Sports Discus), Scopus, ProQuest (Sports Medicine \& Education Index) and the Cochrane Central Register of Controlled Trials (CENTRAL). The search was limited to full-text articles published in English from the inception of the databases until the 19th of January 2021.

The full search strategy is provided in detail as an additional file (see Additional file 1).

\section{Screening process}

The screening process strictly adhered to the 'a priori' objective eligibility criteria elaborated in the PROSPERO protocol. Abstracts and titles retrieved in the search of the electronic databases were exported to EndNote X9 and screened independently by two reviewers ( $\mathrm{HN}$ and $\mathrm{AB})$ to reduce the possibility of rejecting relevant reports (Fig. 1). Only studies that did not clearly match the inclusion criteria were excluded (e.g. populations such as individuals with Parkinson's disease, traumatic brain injury, etc.; study designs such as case studies, cross-sectional studies, etc.; different types of robots, like robots for training the upper extremities, etc.; types of reports such as conference papers, reviews, etc.). All remaining articles advanced to the next step of the screening process and were scrutinized in full-text before potential inclusion. The included articles were divided between two reviewers ( $\mathrm{HN}$ and $\mathrm{AB}$ or $\mathrm{MS}$ ) for independent data extraction. Risk of bias was independently assessed by the same two reviewers using the Cochrane Risk of Bias 2 (RoB 2) tool [32]. Another reviewer (AA) was available to adjudicate any potential disagreements to help reach a consensus.

A meta-analysis using a random-effects model was performed with Review Manager 5 (Copenhagen: The Nordic Cochrane Centre, Cochrane) when a minimum 


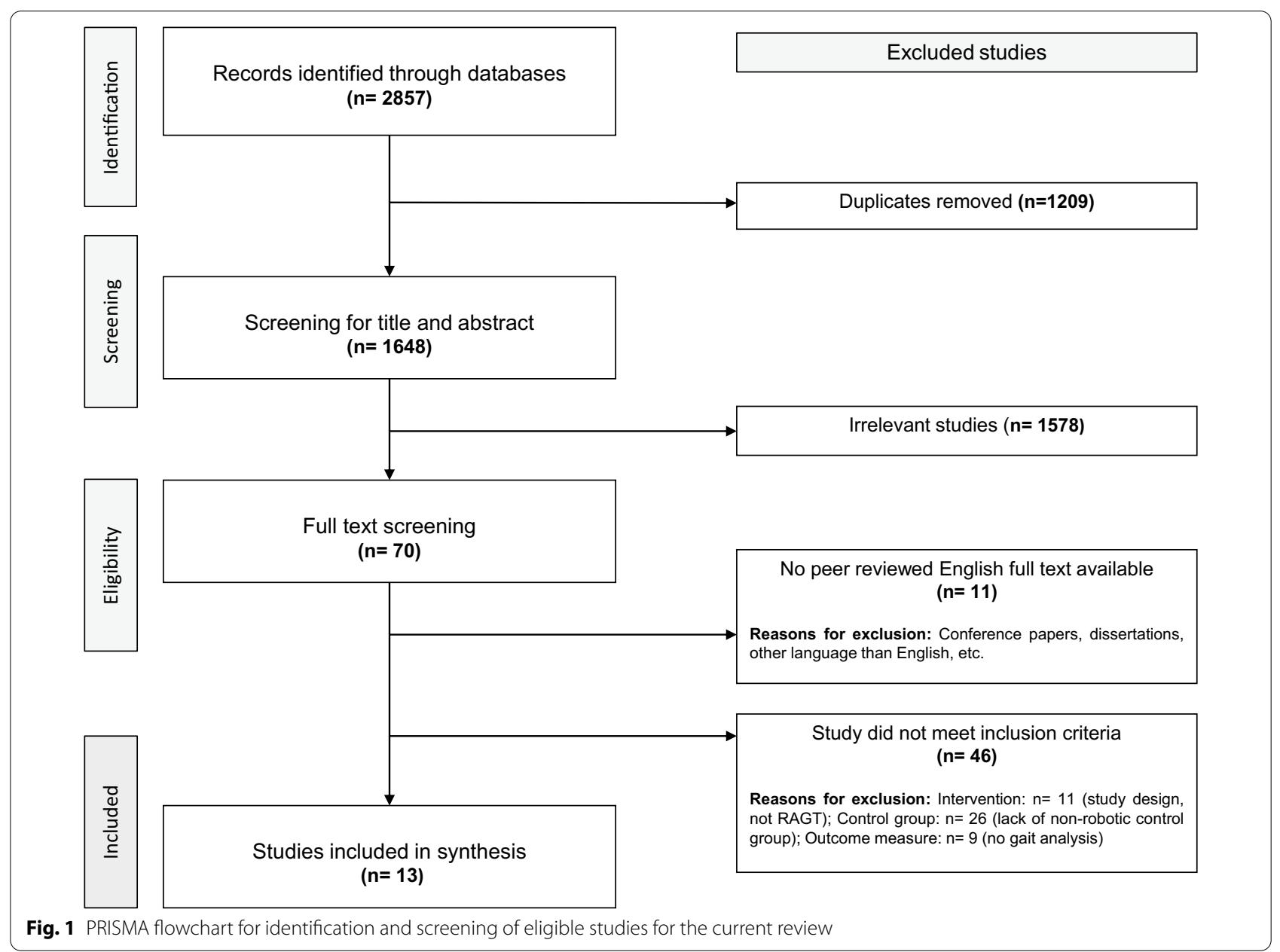

of three studies with relevant data, adequate homogeneity of population, interventions and chosen outcome measures were available. An $\mathrm{I}^{2}$ value $>40 \%$ was considered as the threshold for statistical heterogeneity [33]. Subgroup analyses were performed regarding the effects on gait speed and cadence depending on velocity during the assessment (self-selected, SSV, versus fastest possible, FV), type of RAGT employed in the intervention group ( $\mathrm{t}$-RAGT versus o-RAGT) and time of publication (studies published 2007-2014 versus studies published 2015-2020). When a study had two intervention groups and one comparator group, the data from the intervention groups were pooled (if their findings were identical) in the synthesis. For instance, one study [34] used two intervention groups performing RAGT. One used additional direct transcranial stimulation during RAGT, while the other group received sham transcranial direct stimulation during the same training. The results in these two groups did not differ significantly and were therefore pooled in the meta-analyses.
In addition to the meta-analyses, a descriptive synthesis was performed for the outcomes where statistical pooling was not possible and findings have been presented in a narrative form with complementing tables. The GRADE (Grading of Assessment, Development and Evaluation) criteria [35] were employed to interpret findings and summarise the levels of evidence for both the pooled and narratively summarised data [36]. The evidence was downgraded from "high certainty" by one level for serious (two levels for very serious) concerns about the risk of bias, indirectness of evidence, the inconsistency of findings, imprecision of effect estimates or potential publication bias across studies.

\section{Results}

\section{Characteristics of the included studies}

Of the 2857 studies retrieved, 13 studies involving a total of 412 participants (264 males, 148 females) were eventually included in this review (Table 1). The mean age of the populations in each study ranged from 52 to 69 years. Sample sizes ranged from 12 to 63 participants 







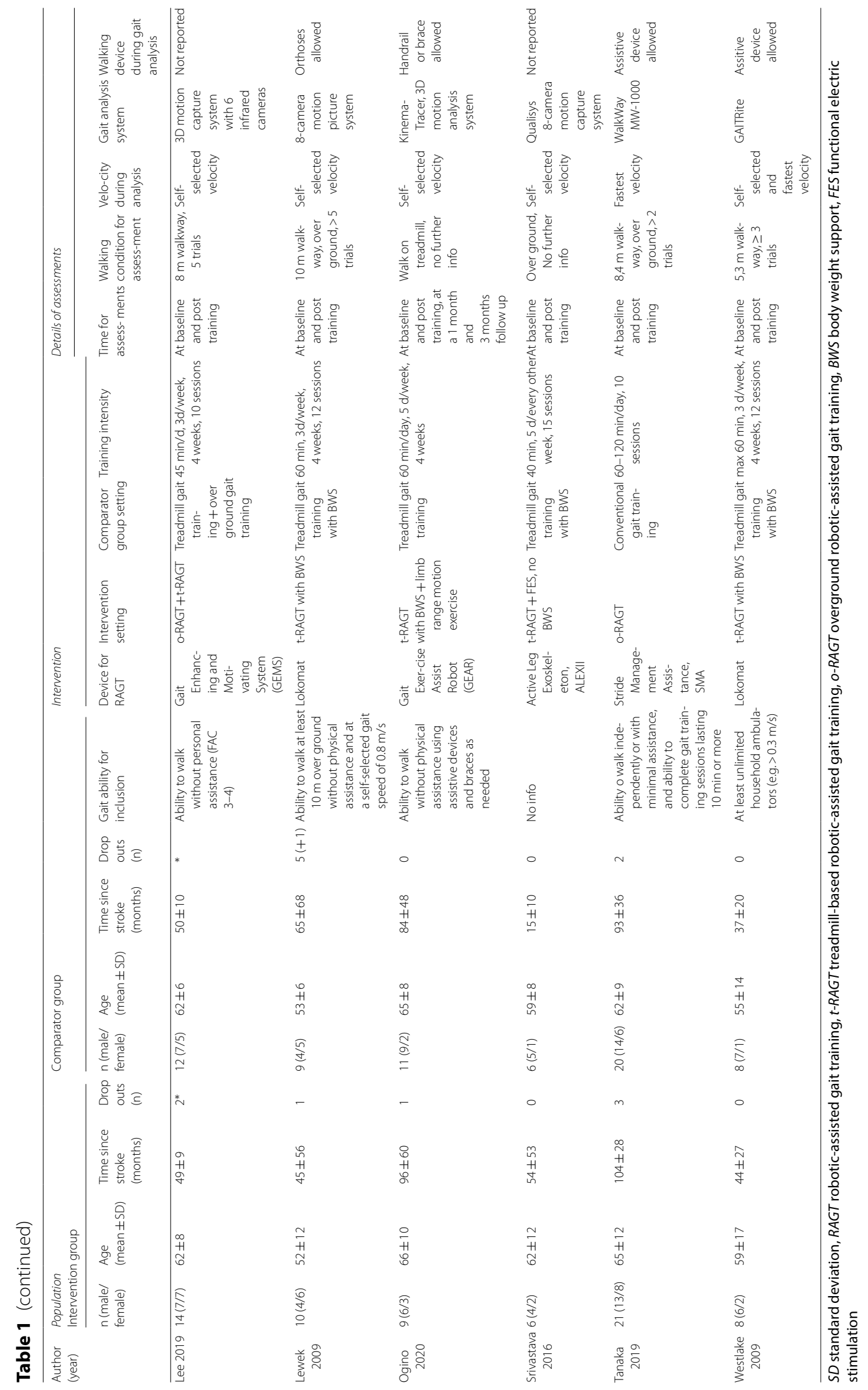


and over half of the studies (62\%) involved sample sizes of 30 participants or less [34, 37-43]. The participants in three of the studies [43-45] were in a subacute phase (mean time up to 4 months post-stroke), while the rest were in a chronic phase post-stroke (mean time up to 104 months post-stroke). Gait ability for inclusion in each study varied from being independent to needing an assistive device and/or personal assistance for walking. The intervention groups received either RAGT as a monotherapy $(n=10)$ [34, 37-40,42, 44-47] or in combination with some kind of non-robotic gait training $(\mathrm{n}=3)$ that was similar to the training received by the comparator group [41, 43, 48]. All studies except one [34] used exoskeletons (Lokomat, GEMS, EKSO, ALEXII, SMA, GEAR) and the majority of these $(n=8)$ employed t-RAGT with body weight support [34, 37, 38, 40, 41, 43, 44, 47]. One study [39] combined t-RAGT (no body weight support) and o-RAGT. The comparator groups of the included studies received conventional gait training [43-45, 48], overground gait training [34] and/or treadmill gait training with [37, $38,40-42,47]$ or without body weight support $[39,46]$ (Table 1).

The duration of the study interventions ranged from 10 days to 10 weeks, yielding $10-40$ sessions, given with a frequency of two to five times per week. Duration ranged from 30 to 105 min per day. Comparator and intervention groups were offered training with similar duration and frequency. Details of the received training (e.g., intensity) were however vague or inadequately reported in many studies. This was specifically true for the training received by the comparator group, where the interpretation of "conventional gait training" or "traditional gait training" may have differed.

The dropout rate during the intervention period ranged from 0 to $37 \%$. In six studies, all included participants completed the training throughout the whole intervention period [34, 38, 40, 42, 46, 48]. Reasons for withdrawal included fear of falling, skin lesions, leg pain due to training, problems with an orthosis, pitting oedema, injury related to training, or self-reported exercise intolerance [37, 43-45, 47]. Dropout was also due to travel limitations and medical or personal reasons that were reported to be unrelated to training [37, 41, 43-45, 47].

All studies performed a baseline and post-intervention assessment within a couple of weeks after the participants completed the training period. In addition, one study [34] included a 2-week follow-up evaluation, while four studies $[41,44,46,47]$ performed follow-up evaluations 1-6 months post-intervention to investigate the long-term effects of RAGT. To collect biomechanical data, gait analysis was performed on a gait mat (GAITRite, Walkway or GaitMat) [34, 38, 40, 44-47], with a 3D motion capture system [38, 39, 41, 42], an accelerometer [48], or an inshoe plantar pressure measurement system [43]. Of the 13 included studies, biomechanical measures were presented as primary outcomes in nine [37-41, 45-47] and as secondary outcomes in four [34, 42-44].

\section{Risk of bias assessment}

A summary of the risk of biases is reported in detail for each included study in Figs. 2 and 3 (generated with Review Manager: Version 5, Copenhagen: The Nordic Cochrane Centre). Risk of bias arising from the randomization process revealed concerns for two studies, either due to a lack of relevant information [42] or that randomization was based on the hospital record numbers of the participants [45] (Fig. 3). Risk of bias due to missing outcome data gave rise to concerns in several studies. Reasons for withdrawal were not reported in one study [39], and when reported they were likely to be related to certain consequences of the training, e.g., fear of falling, skin lesions, leg pain due to training, pitting oedema or self-reported exercise intolerance [37, 43-45, 47]. In all of these studies except for one [43], the dropouts were excluded from the analysis. Selective reporting of results raised some concerns in most of the included studies due to the absence of study protocols or pre-specified analysis plans. Only four studies reported trial registrations $[39,41,46,48]$. The analysis plan in one of the registered protocols did not conform to the analyses performed in the study [48], and one study [41] chose to report only within-group analyses.

\section{Effect on temporal and spatial parameters}

Although all included studies analysed at least one temporal gait parameter, the most reported were gait speed $(n=10)$ and cadence $(n=8)$. The certainty of evidence (GRADE evaluation) was found to be very low for both these parameters because there were some concerns for several studies with regard to the risk of bias (Table 2), indirectness of evidence owing to clinical variation regarding intervention parameters and gait analysis settings (see Table 1), and imprecise findings with insignificant differences between small population groups (6-25 participants/group). The meta-analysis of gait speed (Fig. 4) indicated no overall significant differences between the intervention and comparator groups after the training period (mean difference [MD] $0.00 \mathrm{~m} / \mathrm{s}$; $95 \%$ confidence interval $\left.[\mathrm{CI}]-0.05,0.05 ; \mathrm{I}^{2}=93 \%\right)$. The meta-analysis of cadence similarly did not reveal any significant differences between groups (MD 1.44 steps/min; 95\% CI - 2.34, 5.22; $\mathrm{I}^{2}=92 \%$ ) (Fig. 5). Bang et al. [38] did not report gait velocity during assessment (SSV or FV) and this study was therefore excluded from the subgroup 
meta-analyses. This study found significantly larger improvements for both gait speed (MD $2.14 \mathrm{~m} / \mathrm{s} ; 95 \% \mathrm{CI}$ $0.93,3.36$ ) and cadence (MD 1.48 steps $/ \mathrm{min} ; 95 \%$ CI 0.41 , 2.55) favouring the RAGT group. When these results were included in the meta-analyses, they did however not influence the overall results.

In the subgroup analyses of gait speed and cadence (see Additional files 2 and 3), where the studies employing o-RAGT and those using t-RAGT were differentiated, we did not find any between-group differences. Differences between groups were neither identified in subgroup analyses that differentiated between earlier (2007-2014) and later published studies (2015-2020) with regards to gait speed and cadence (see Additional files 4 and 5).

Other temporal parameters assessed in the included studies were gait cycle/stride duration [43, 48], step time [46], stance time/percentage of GC (single [43, 47] and/ or double limb support [38,41,43]), and swing time/ percentage of GC [41, 46] (Table 3). For the same reasons as for the outcomes used in the meta-analysis, the GRADE assessment indicated very low certainty of evidence for the gathered temporal parameters (Table 2). However, in nearly all studies, no significant difference in temporal parameters between groups was observed and both groups improved to an equal extent. A significantly higher increase in the single-limb stance period favouring the comparator group during FV was reported by Hornby et al. [47] (MD - 3.0\%; 95\% CI - 6.96, 0.96) (Table 3). In contrast, Bang et al. [38] observed a decreased double limb support time (MD - 1.46\%; 95\% CI - 2.32, 0.6) favouring the RAGT group during walking in SSV. Although Calabró et al. [48] reported a significantly higher effect for gait cycle duration in the RAGT group, our calculations (based on measurements of distances in the pdf-file and calculations using RevMan) did not reveal any significant difference between the groups (MD - 0.08 (ratio); 95\% CI - 0.19, 0.03) (cf. Tables 3). Finally, the study by Ogino et al. [41] that did not report results of between-group analyses was excluded in the narrative synthesis.

Among spatial parameters, step length increased significantly more on the affected side following RAGT compared with non-robotic gait training (MD $2.67 \mathrm{~cm}$; 95\% CI 1.55, 3.80; $\mathrm{I}^{2}=65 \%$ ) (Fig. 6). Such between-group differences were not detectable for step length on the non-affected side, nor for the combined (affected and non-affected side) change. As seen in Fig. 7, stride length increased significantly more in the RAGT group when compared with the comparator group (MD $2.88 \mathrm{~cm}$; 95\% CI $0.46,5.25 ; \mathrm{I}^{2}=66 \%$ ). However, the (GRADE) certainty of evidence remained very low for both these parameters (Table 2).
Seven studies $[34,37,39,40,45,46,48]$ calculated some kind of temporal or spatial symmetry ratio (Table 4) by using a variety of ratio calculations of different spatiotemporal parameters for either or both the paretic and non-paretic limbs. Results from the meta-analysis of temporal symmetry (Fig. 8) revealed very low evidence (Table 2) for a statistically significant improvement in the symmetry ratio in the RAGT groups compared with the non-robotic gait training groups (MD 0.09; 95\% CI $0.04,0.15 ; \mathrm{I}^{2}=90 \%$ ). For spatial asymmetry (Fig. 9) on the other hand, no significant differences were observed between groups ( $\mathrm{MD}-0.01 ; 95 \% \mathrm{CI}-0.06,0.04 ; \mathrm{I}^{2}=80$ ).

\section{Effect on kinematic parameters}

Only three of the included studies [37, 41, 42] analysed kinematic data, and the overall (GRADE) certainty of evidence was found to be low for the kinematic parameters (Table 4). Lewek et al. [37] detected the consistency of intra-limb hip and knee angular trajectories over repeated gait cycles and the maximum lateral deviation of the heel during the swing phase with respect to the position of the ipsilateral heel during consecutive stance phases (known as circumduction). Srivastava et al. [42] analysed the peak flexion angles during the swing phase. Both studies analysed gait at SSV but none of them found any differences between groups after the intervention period. Ogino et al. [41] used kinematic data to detect index values for abnormal gait patterns following stroke. However, they did not report results from between-group analyses.

\section{Effect on kinetic parameters}

Kinetic variables represent the forces generating the kinematics and spatiotemporal outcomes during walking, and kinetic information should therefore be very useful for understanding and interpreting gait characteristics [49]. However, we did not find any eligible RCTs evaluating kinetic gait data following RAGT.

\section{Follow-up data}

Four studies performed a follow-up test: one after 2 weeks [34], one after 1 month [41], three after 3 months $[41,44,46]$ and one after 6 months [47]. The outcome measures investigated during the follow-up period were gait speed, cadence, step time, swing/stance time, step length, temporal and spatial symmetry and gait kinematics (Tables 3 and 4). The 2 week follow-up by Geroin et al. [34], found similar group differences in cadence and temporal symmetry that had been observed during the assessments immediately after the intervention period. According to this, the RAGT group showed a significant improvement in cadence (MD 15.6 steps $/ \mathrm{min}$; 95\% CI $8.15,23.11$ ) and temporal symmetry (MD -0.42 (ratio); 


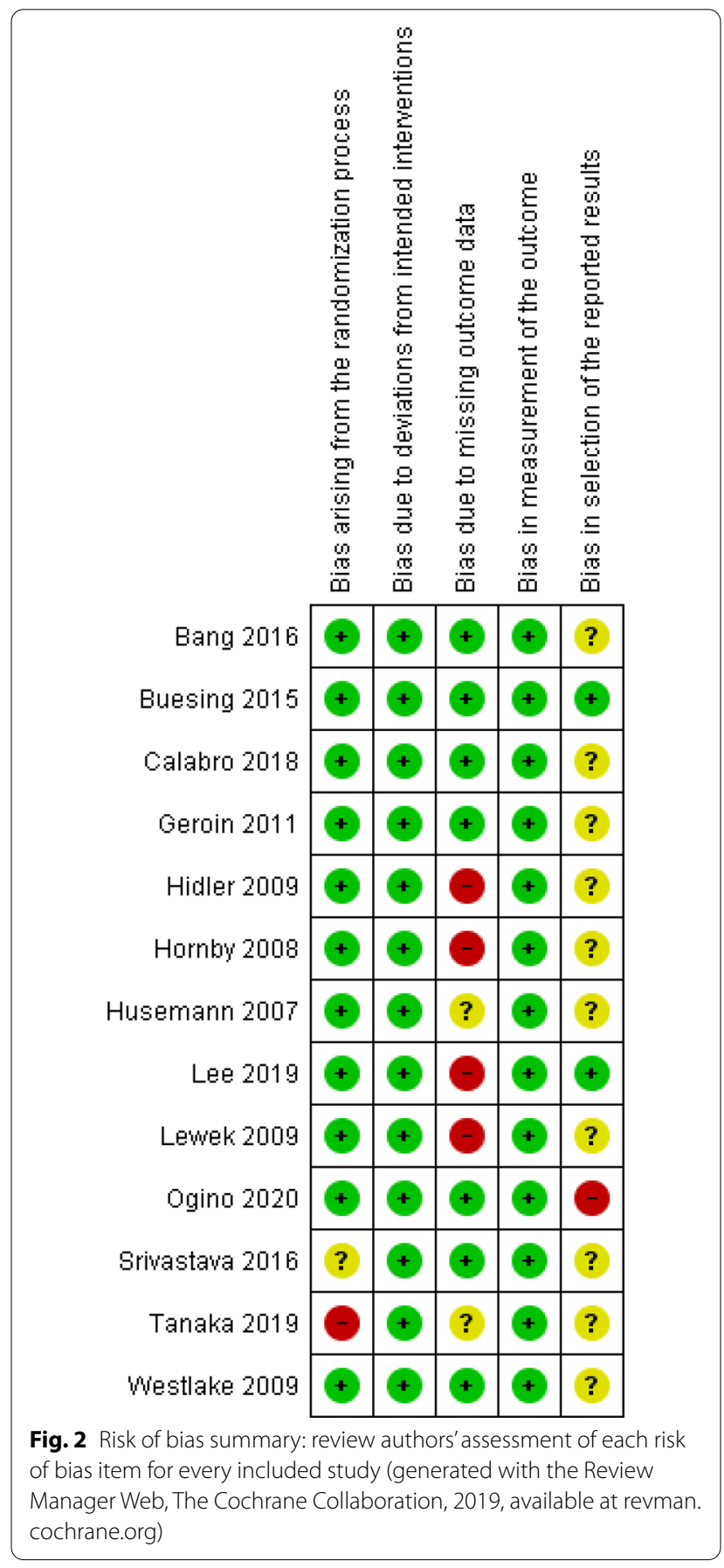

95\% CI $-0.5,-0.34)$ when compared with the comparator group. No group differences were otherwise detected for any of the investigated outcomes when RAGT was compared with non-robotic gait training during followup measurements $[44,46,47]$.

\section{Discussion}

Except for synthesised evaluations of gait speed, this is the first study to review and ascertain the level of evidence for RAGT with the quantification of post-stroke gait quality based on biomechanical measures. While employing a pre-registered comprehensive search strategy, based on well-defined eligibility criteria for studies, in nine renowned databases and screening 2857 retrieved citations, only 13 RCTs met the eligibility criteria to address the research question.

Analysis and synthesis of the included studies revealed mixed effects on biomechanical measures assessed after RAGT. Risk of bias assessment raised concerns for several of the studies due to limitations in the randomization process and poor reporting regarding the handling of missing data (See Figs. 2 and 3). Furthermore, reporting bias could be a problem as the plan for analysis was seldom available and only three studies $[39,46,48]$ reported a registered study protocol. The variety of outcome measures used in the included studies limited the ability to pool results. However, meta-analyses for gait speed and cadence showed no effect of RAGT that exceeded the effect of non-robotic treatment (Figs. 4 and 5). Owing to the uncertainty of the evidence, specifically concerning the risk of bias, small population sizes, and heterogeneity and inconsistency in results, the general quality of evidence for these outcomes was downgraded to very low despite including only randomized controlled trials (RCTs) (Table 2). The low number of eligible RCTs identified for inclusion in this review, in combination with the concerning risk of bias associated with them, rendered a low certainty of evidence for the effects of RAGT on gait speed among persons with stroke compared with non-robotic training. Methodologically robust RCTs are required to elucidate any potential effects of RAGT on biomechanical parameters relating to post-stroke gait.

Evidence for the potential of RAGT, in combination with physiotherapy, to increase the likelihood of regaining independent walking ability post-stroke has previously been reported by Mehrholtz et al. to be moderate [12]. Their findings, as well as the findings of yet another review [14], were in line with ours and showed that gait speed (assessed either with clinical or instrumented methods) improved to an equal extent in the RAGT and comparator groups post-stroke.

We found the certainty of evidence for the effect of RAGT on cadence to be very low for the same reasons as mentioned for gait speed (Table 2). The pooled results revealed an equal amount of improvement in cadence in intervention and comparator groups (Fig. 5). Gait speed 
Bias arising from the randomization process

Bias due to deviations from intended interventions

Bias due to missing outcome data

Bias in measurement of the outcome

Bias in selection of the reported results
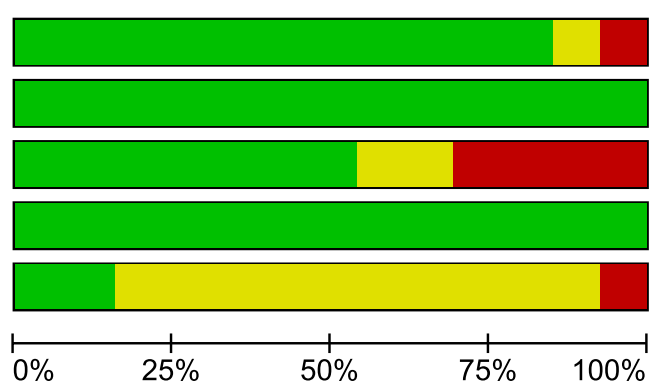

\begin{tabular}{|ll}
\hline Low risk of bias $\quad \square$ Some concerns $\quad \square$ High risk of bias \\
\hline
\end{tabular}

Fig. 3 Risk of bias graph (generated with the Review Manager Web, The Cochrane Collaboration, 2019, available at revman.cochrane.org)

and cadence on their own do not reflect the specific gait movement pattern during walking and should instead be interpreted in combination with other spatial and/or temporal kinematic parameters $[21,50]$. Step length and stride length are considered closely connected to gait speed [74]. However, even though a difference between groups was not detectable with regards to gait speed, our analyses showed that step length (Fig. 6) and stride length (Fig. 7) on the affected side improved more in the RAGT group when compared with the non-robotic training group.

The typical motor impairments following stroke cause an unbalanced walking pattern which is often characterized by asymmetries in temporal parameters (relation between affected and non-affected swing, stance, step and/or stride time) and/or spatial parameters (relation between affected and non-affected limb positions and/or step length) $[51,52]$. The consequences of spatial and temporal asymmetry are debated [53], but symmetry ratios seem to be more sensitive measures of recovery compared with absolute values of spatial or temporal parameters [54]. Gait symmetry is further associated with a high energy expenditure [55], a high risk of falls, and an unequal loading of the joints (increasing the risk of joint degeneration and bone density loss in the paretic limb) [56]. Our meta-analysis showed that temporal symmetry improved slightly more in the RAGT groups when compared with the non-robotic gait training groups (Fig. 8). There were conversely no significant differences observed between groups with regard to spatial symmetry after training (Fig. 9). The pattern of spatial symmetry vary greatly, while some individuals exhibit a longer step length on the non-paretic side, and others take longer steps on the paretic side [57, 58]. A step lengthening strategy on the affected side detected for the RAGT group (see Fig. 6) may not necessarily be associated with a similar improvement in spatial symmetry in the same group. The level of asymmetry is suggested to be more relevant than some other parameters (e.g., gait speed and step length) to identify the degree of impairment and compensatory mechanisms used during walking post-stroke [59]. These results should however be interpreted cautiously since the GRADE certainty of evidence was very low due to inconclusive results between studies and since there were only a few studies with relatively small sample sizes that investigated this.

Meta-analyses that differentiated studies from before and after 2015 found no differences between the groups with regards to gait speed and cadence (see Additional files 4 and 5). However, when contrasting the findings of most recent studies with those of earlier research (Tables 3 and 4), the most recent studies (published from 2018 to 2019) reported larger improvements of spatial and temporal parameters in the RAGT groups when compared with the non-robotic training groups $[39,45,48]$. This may reflect how increased knowledge in the area of gait rehabilitation post-stroke, combined with the rapid technological development of advanced robotic devices, may have improved RAGT post-stroke. Several factors are considered imperative to stimulate increased neural activity and reorganization: (1) enhancement of active wearer participation, which includes the possibility of individual adjustments and an adaptable robotic interference [3, 8, 60, 61], (2) sufficient degrees of freedom to minimize motion restrictions in the joints and allow other aspects of gait, such as balance, to be incorporated in training [3, $8,60,61]$, and (3) real-time biofeedback to the wearer [8]. The trend towards more positive results for the biomechanical gait measures in the most recent studies evaluating RAGT post-stroke might be a result of adjustments made based on the factors mentioned above. For instance, Lee et al. (2019) [39] and Calabrò et al. (2018) [48] provided active wearer participation through individual guiding, encouragement, adaptation and progression. 


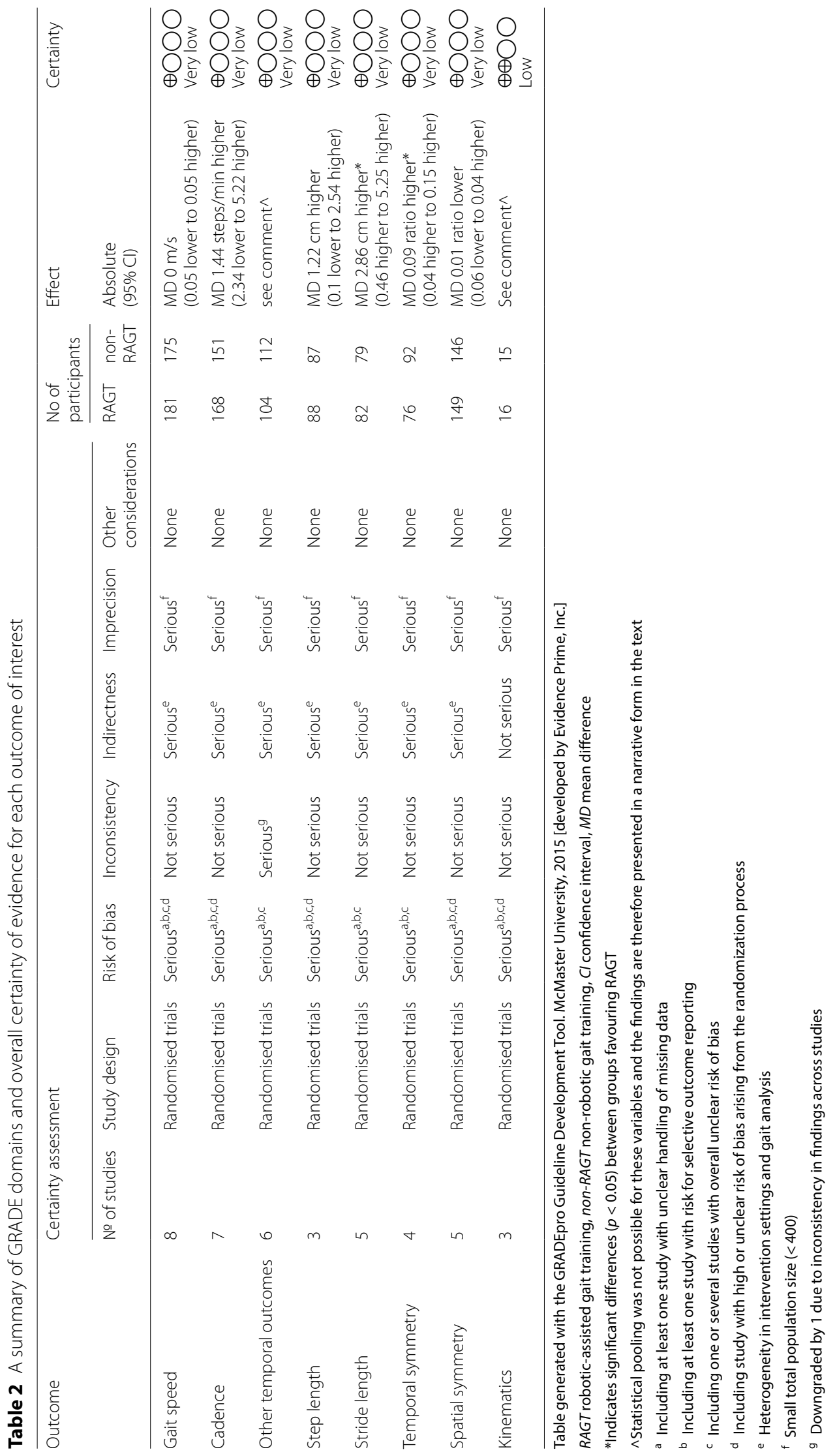




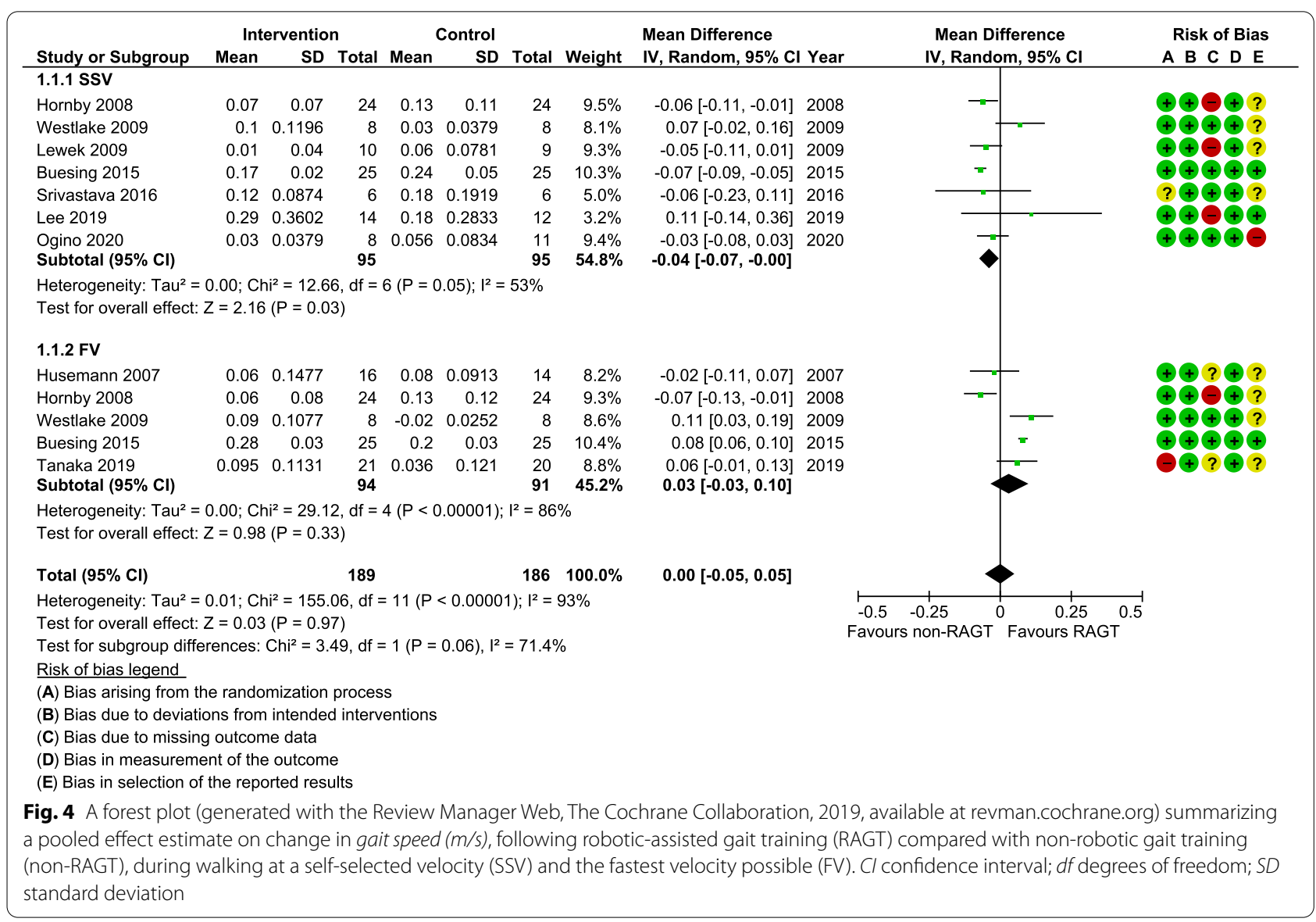

Low certainty of evidence indicated that kinematic measures were not significantly different between the groups after the intervention [37, 42]. Although kinematic measurements could help to discriminate between restoration and compensation strategies that develop post-stroke, only two studies [37, 42] that collected kinematic data after RAGT (Tables 3 and 4) were found. Hence, no certain conclusions can be made regarding the effect of RAGT on kinematic measures of gait post-stroke at this time. It has been advocated that detailed kinematic data should be collected and analysed for quantification of specific gait movement patterns when investigating walking post-stroke $[18,20]$. Even so, the need for sophisticated laboratory equipment, competence of assessors, and more time and finances may be some reasons for the lack of studies that have performed these assessments.

The requirements in terms of finance, assessor competence and time are similar for the collection of kinetic and kinematic data. To obtain valid kinetic data representing joint moments and power, the individual also needs to walk independently without walking aids, as naturally as possible, and contact the force platform with only one foot. This may be a challenging task for individuals poststroke who have severe impairments. We did not find any eligible RCTs that evaluated kinetic variables after RAGT. Spatial and temporal parameters during walking are nevertheless direct consequences of the kinetic parameters affecting the joints [49]. Several studies have shown kinetic deviations in both the affected and unaffected leg in individuals post-stroke [62,63]. Moments and power bursts are suggested to be reduced in amplitude in persons post-stroke when compared with asymptomatic controls while walking at a self-selected speed [64]. However, the total lack of eligible robust RCTs evaluating kinetic variables after RAGT post-stroke calls for future research in this area.

\section{Methodological considerations}

This review followed a pre-registered protocol in PROSPERO, included relevant RCTs published until the 19th of January 2021, employed the updated and comprehensive Cochrane risk of bias 2 tool (2019), and summarized the current level of evidence for the biomechanical gait parameters of interest using the established GRADE criteria. In general, the RAGT and comparator groups were 


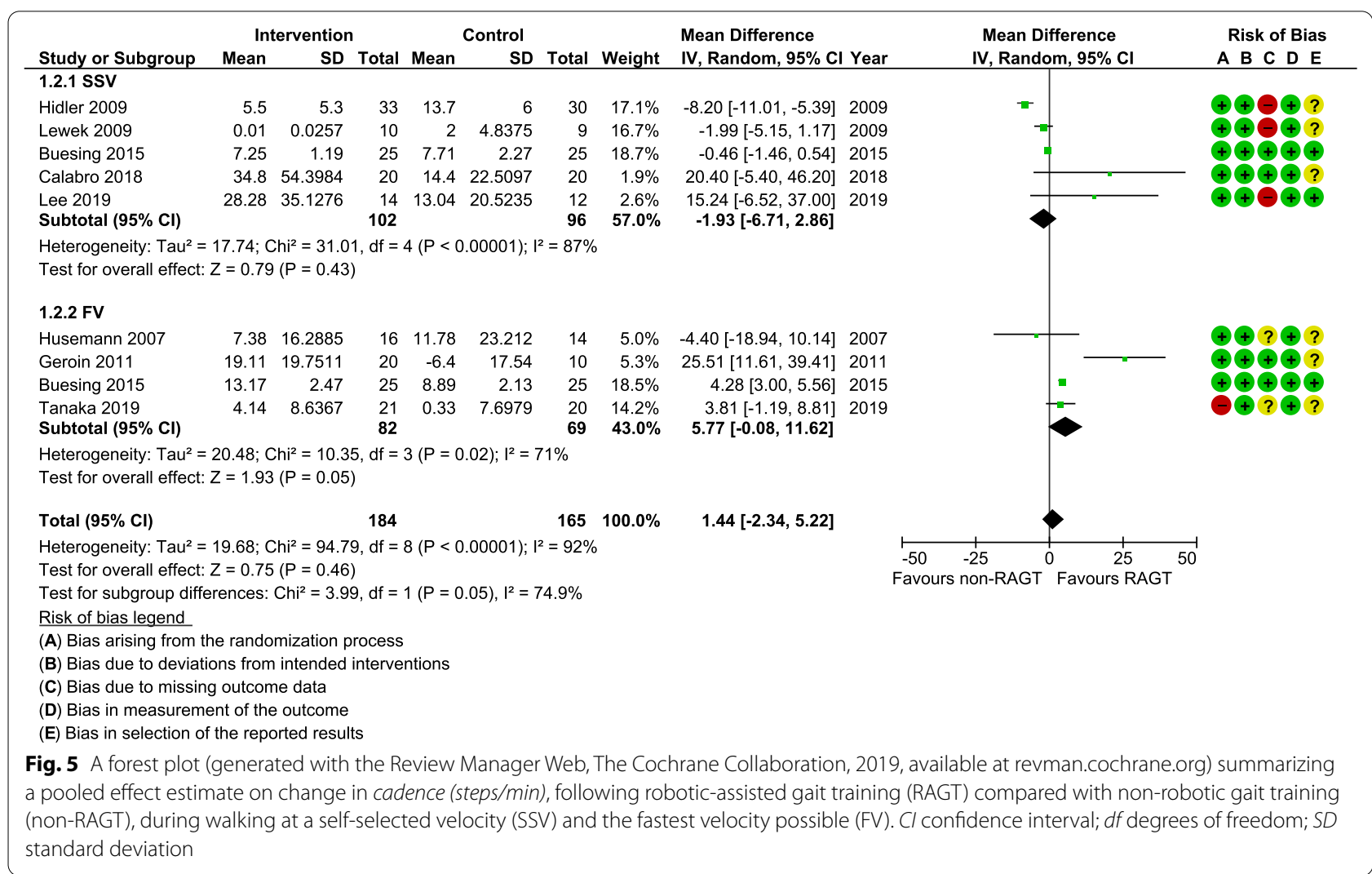

comparable based on the amount of therapy (duration and frequency) provided, but information on the intensity (here defined as the amount of work per unit time [i.e., the rate of work or power]) of training and individual adjustments was inadequate or unavailable. The importance of specifying the intensity of practice has been emphasized [65]. The intensity is nonetheless applied and specified inconsistently in most exercise training protocols. This is also the case for the studies included in this review, and this may account for some of the differences in the conclusions regarding the lack of superiority of one rehabilitation method compared with the other. Clinical heterogeneity between the included studies was also considerable due to differences in the population of interest (stroke severity level and phase post-stroke, etc.) and intervention settings (the robotic device used, feedback delivered, and content, duration, intensity and frequency of the training in both the RAGT as well as the comparator groups).

The chosen biomechanical outcome measures (different spatial and/or temporal parameters) and the settings for gathering gait data varied (various systems for gait analysis, allowance of walking aids during assessments, variations in walking distances, etc.). This variation together with different definitions and/or calculations in data analysis limit the possibility of generalising the results. We also did not include studies using only electromyography (EMG) in our review because motor intent identification using EMG may have significant limitations in individuals post-stroke due to severe motor impairment, profound muscle fatigue, or abnormally coactivated muscles [66,67]. This is further corroborated in an up-to-date compilation of evidence in this area provided by Lennon et al. (2020) [68]. Finally, this review excluded papers in languages other than English.

It has been suggested that the effect of RAGT depends on factors such as time after stroke and impairment severity [12]. Indeed, RAGT combined with physiotherapy has been suggested to be especially efficient in improving the function and mobility of the lower limbs in nonambulatory patients in their subacute phase post-stroke $[11,13]$. It has also been hypothesized that gait function and movement pattern is less likely to change in a chronic phase post-stroke. Interventions for improving motor performance, initiated in an early stage post-stroke, are assumed to closely interact with the dynamic phases of neural remodelling to promote better reorganization [69]. Hence, the possibility to influence the gait movement pattern post-stroke would supposedly decrease with the course of time post-stroke. Only three of the thirteen 


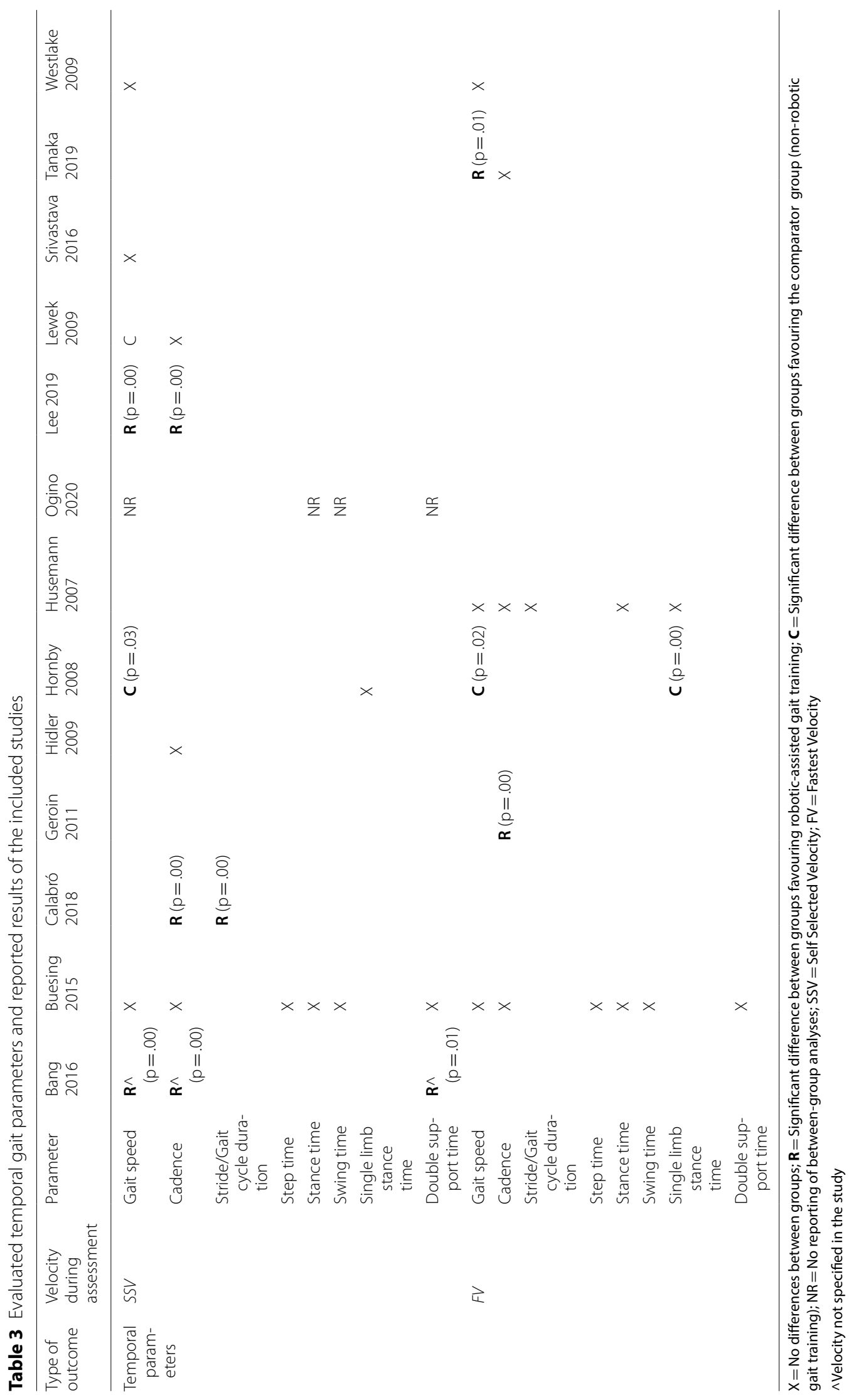




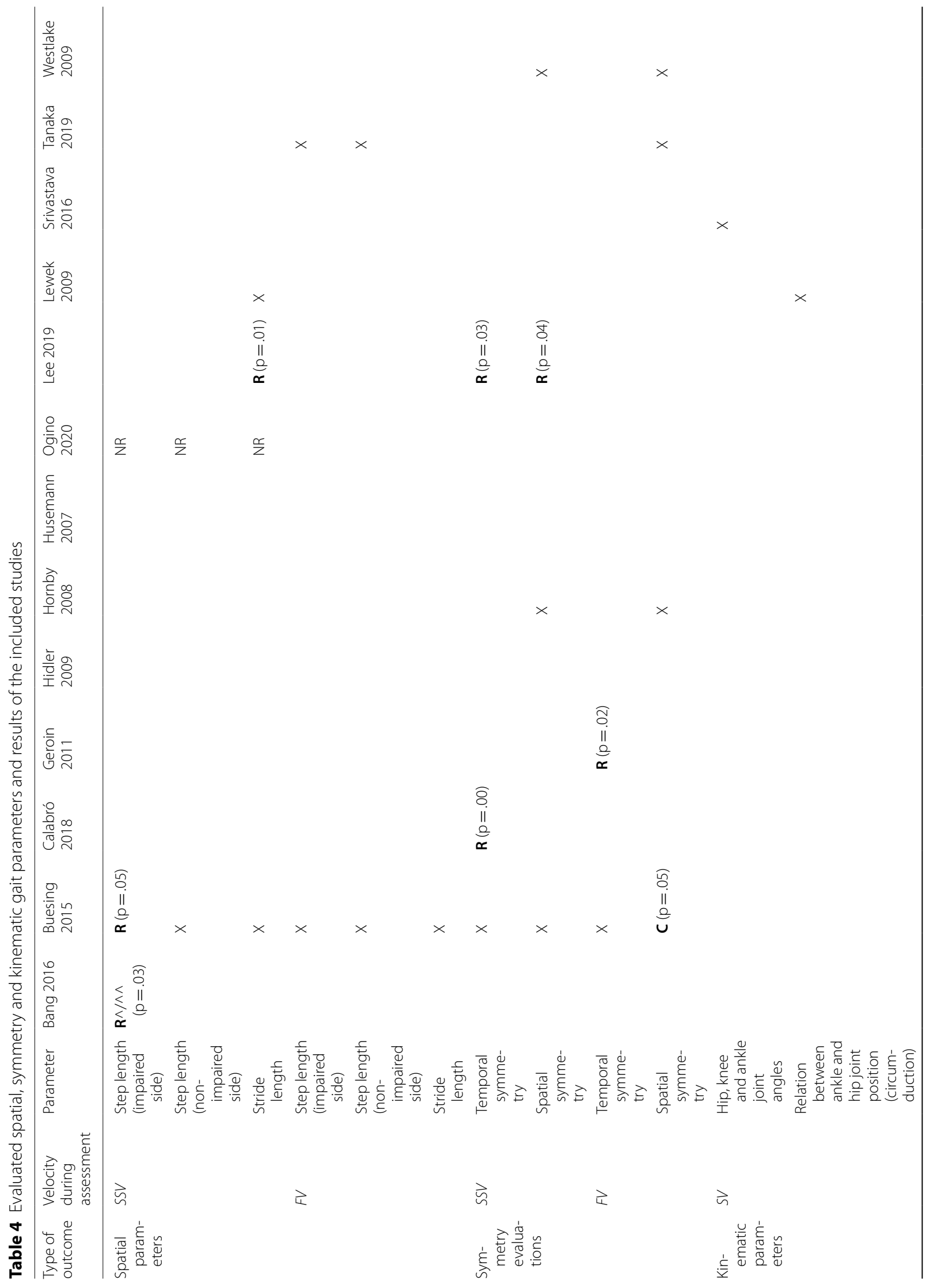




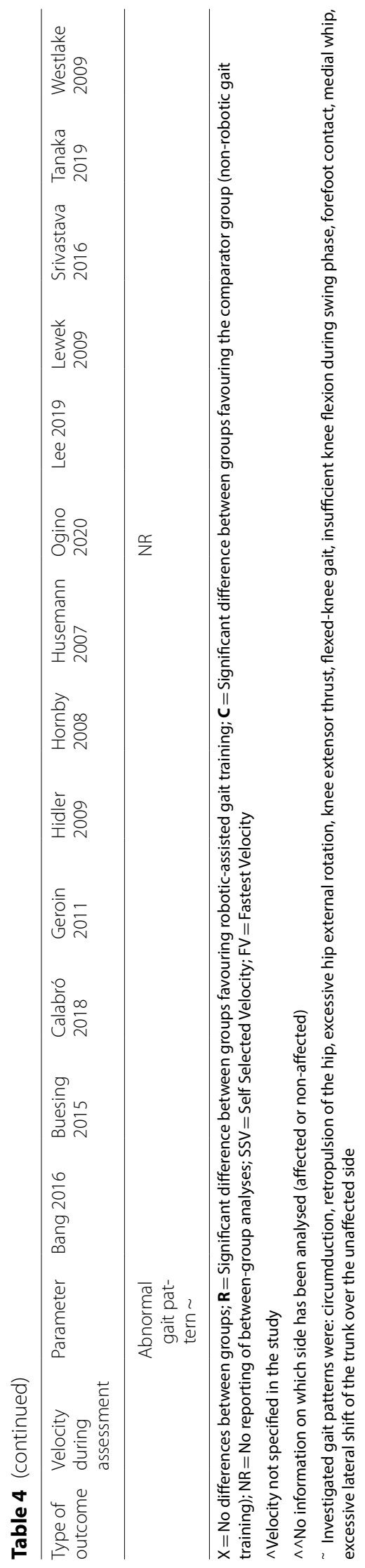




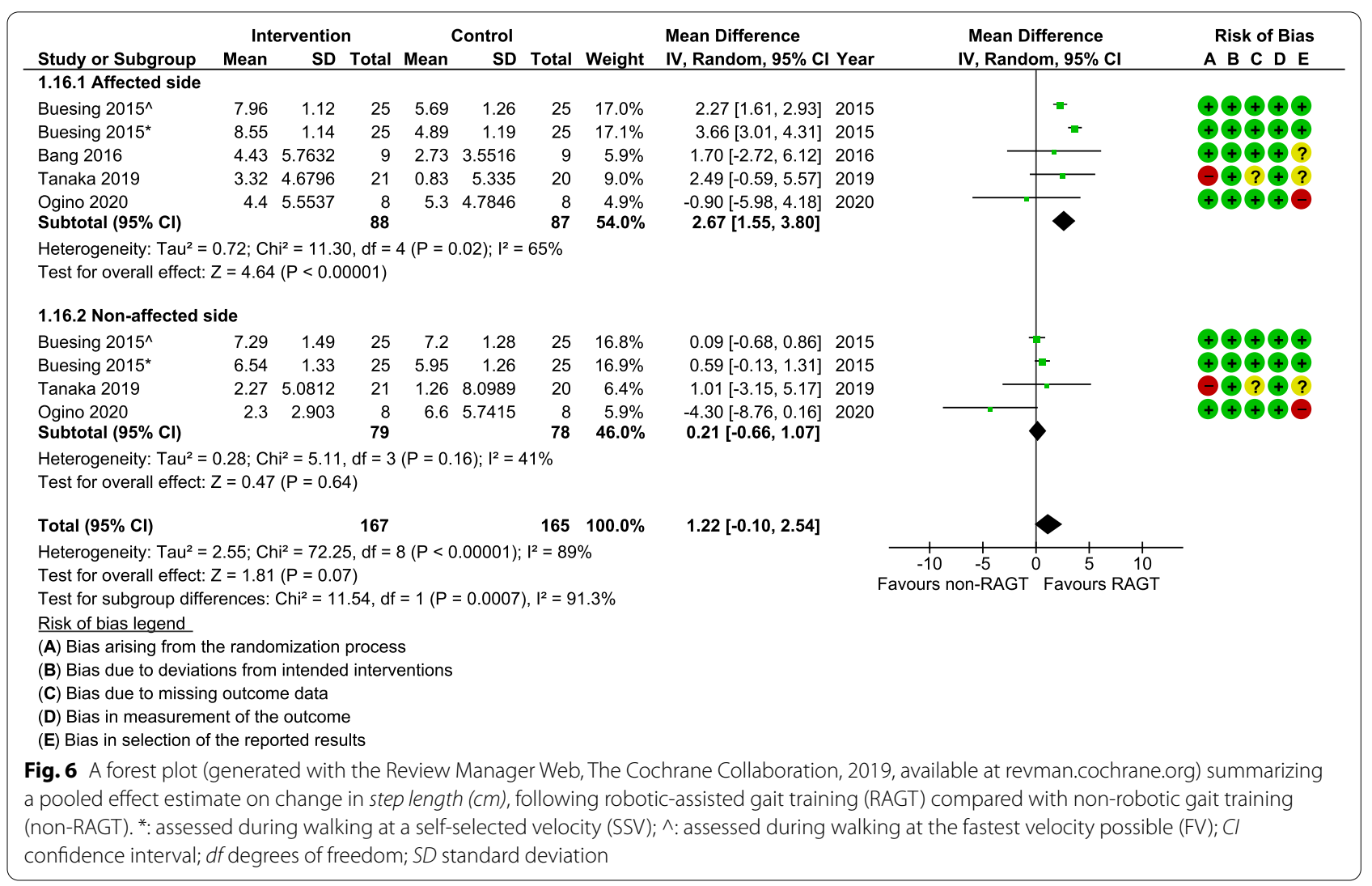

studies included in this review had a population in a subacute phase post-stroke, whereas the others addressed populations in a chronic phase. Since the assessment of gait biomechanics requires walking function (with or without aids or personal support), none of the studies included non-ambulatory persons. It was thus not possible to draw any conclusions regarding the impact of RAGT in relation to the different phases post-stroke (subacute/chronic) or different severity levels of impairments.

Another topic of interest when evaluating the effects of RAGT is the possible difference between RAGT employed on a treadmill and the one performed overground. Overground RAGT is hypothesized to provide greater motor control stimulation, multisensory plasticity and required effort when compared with RAGT performed on a treadmill [5]. Hence, we performed subgroup-analyses based on the employed type of RAGT for the outcomes of gait-speed and cadence but found no significant differences between the groups (Fig. 6).

\section{Future challenges and recommendations}

Robotics in gait training post-stroke requires an evaluation from several perspectives in order to identify responders and non-responders to RAGT (e.g., the impact of RAGT in relation to the different phases poststroke and different severity levels of impairments) and map the strengths and weaknesses to support and guide future technical development. Highly intensive, taskspecific and repetitive gait training post-stroke, such as RAGT, is assumed to stimulate restoration of motor skills and, consequently, normalize the gait movement pattern through neuroplasticity [70-72]. Yet this has not been thoroughly investigated and the mechanisms underlying functional gains achieved through RAGT in individuals post-stroke are still poorly understood. This review highlights the need to combine the measures of task accomplishment with objective assessments of gait movement patterns and gait quality after RAGT.

A consensus is unfortunately lacking as to which biomechanical gait measures to use when investigating motor coordination and the quality of movement patterns during walking $[50,73,74]$. Standardised guidelines for assessing and reporting gait variables should be developed to support researchers and enable pooling of results to facilitate the evaluation of the effects of and further development of gait-assisting robots used in post-stroke rehabilitation. These guidelines should include assessments of several (spatial, temporal and kinetic) aspects of gait $[15,50]$, and consider bilateral motor coordination 


\begin{tabular}{|c|c|c|c|c|c|c|c|c|c|c|c|}
\hline \multirow[b]{2}{*}{ Study or Subgroup } & \multicolumn{3}{|c|}{ Intervention } & \multicolumn{3}{|c|}{ Control } & \multicolumn{2}{|r|}{ Mean Difference } & \multirow{2}{*}{\multicolumn{2}{|c|}{$\begin{array}{l}\text { Mean Difference } \\
\text { IV, Random, } 95 \% \mathrm{Cl}\end{array}$}} & \multirow{7}{*}{ 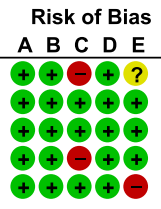 } \\
\hline & Mean & SD & Total & Mean & SD & Total & Weight & IV, Random, $95 \% \mathrm{Cl}$ Year & & & \\
\hline Lewek 2009 & 5 & 12.5673 & 10 & 3 & 7.0903 & 9 & $6.1 \%$ & $2.00[-7.06,11.06] 2009$ & & & \\
\hline Buesing $2015^{*}$ & 15.31 & 2.15 & 25 & 10.61 & 2.28 & 25 & $43.3 \%$ & $4.70[3.47,5.93] 2015$ & & - & \\
\hline Buesing $2015^{\wedge}$ & 15.17 & 2.22 & 25 & 12.9 & 2.48 & 25 & $42.6 \%$ & $2.27[0.97,3.57] 2015$ & & $\square$ & \\
\hline Lee 2019 & 32.1 & 39.8726 & 14 & 19.49 & 28.9997 & 12 & $0.8 \%$ & $12.61[-13.95,39.17] 2019$ & & & \\
\hline Ogino 2020 & 6.8 & 8.5829 & 8 & 11.9 & 8.2534 & 8 & $7.2 \%$ & $-5.10[-13.35,3.15] 2020$ & & - & \\
\hline Total $(95 \% \mathrm{Cl})$ & & & 82 & & & 79 & $100.0 \%$ & $2.86[0.46,5.25]$ & & $\Delta$ & \\
\hline \multicolumn{7}{|c|}{$\begin{array}{l}\text { Heterogeneity: } \mathrm{Tau}^{2}=3.05 ; \mathrm{Chi}^{2}=11.80, \mathrm{df}=4(\mathrm{P}=0.02) ; 1^{2}=66 \% \\
\text { Test for overall effect: } Z=2.34(P=0.02)\end{array}$} & & & $\begin{array}{cc}-20 & -10 \\
\text { Favours non-RAGT }\end{array}$ & $\begin{array}{l}10 \\
\text { Favours RA }\end{array}$ & \\
\hline $\begin{array}{l}\text { Risk of bias legend } \\
\text { (A) Bias arising from t } \\
\text { (B) Bias due to deviat } \\
\text { (C) Bias due to missin } \\
\text { (D) Bias in measurem } \\
\text { (E) Bias in selection o }\end{array}$ & $\begin{array}{l}\text { he randor } \\
\text { ions from } \\
\text { ig outcom } \\
\text { ent of the } \\
\text { f the repo }\end{array}$ & $\begin{array}{l}\text { mization pr } \\
\text { intended ir } \\
\text { ne data } \\
\text { e outcome } \\
\text { orted results }\end{array}$ & $\begin{array}{l}\text { rocess } \\
\text { interven } \\
\text { s }\end{array}$ & ntions & & & & & & & \\
\hline \multicolumn{12}{|c|}{$\begin{array}{l}\text { Fig. } 7 \text { A forest plot (generated with the Review Manager Web, The Cochrane Collaboration, 2019, available at revman.cochrane.org) summarizing } \\
\text { a pooled effect estimate on change in stride length }(\mathrm{cm}) \text {, following robotic-assisted gait training (RAGT) compared with non-robotic gait training } \\
\text { (non-RAGT). *: assessed during walking at a self-selected velocity SSV; } \wedge \text { : assessed during walking at the fastest velocity possible FV; Cl confidence } \\
\text { interval; } d f \text { degrees of freedom; SD standard deviation }\end{array}$} \\
\hline
\end{tabular}

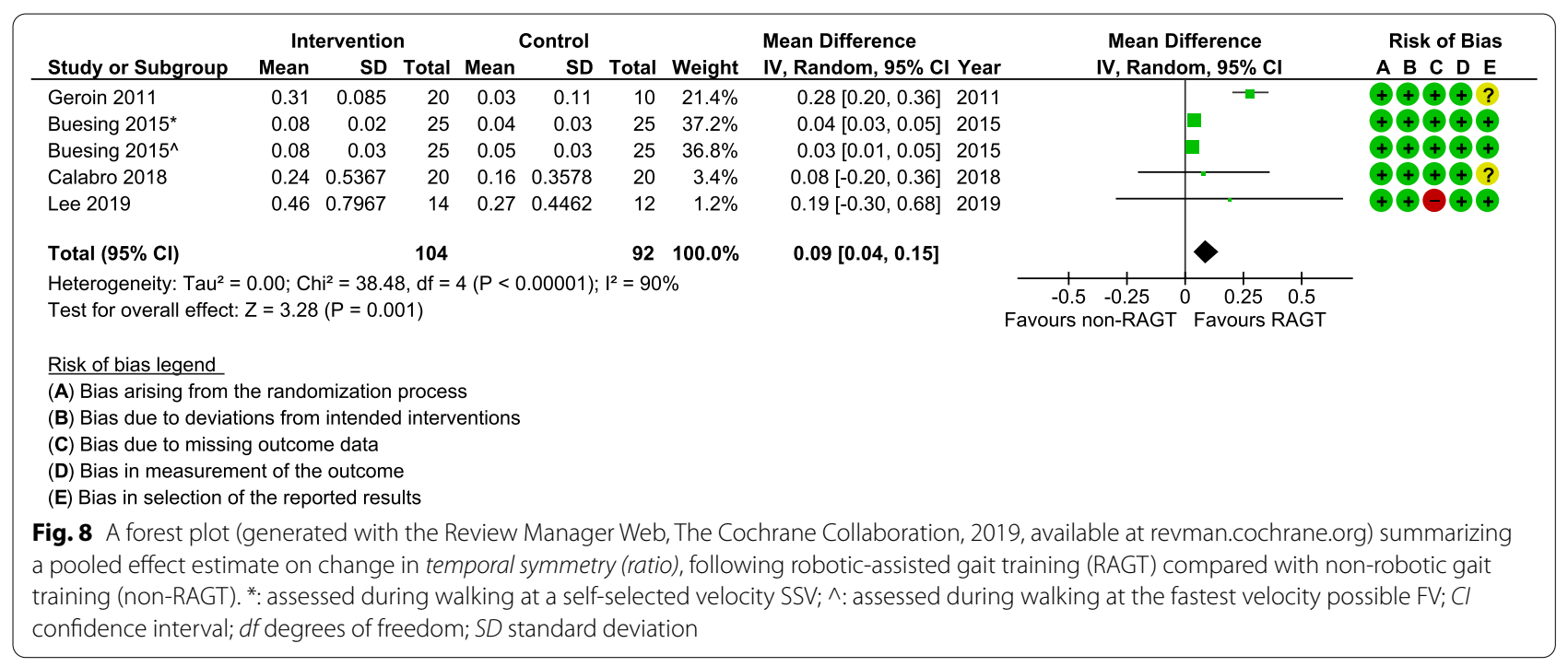

[50] as well as the engagement of the trunk [75] and displacement of centre of mass [76]. Inter-limb coordination, such as symmetry in spatio-temporal parameters, including kinematic measures of movement endpoint, whole trajectories, and joint angles, as well as in kinetic parameters, are all important outcomes reflecting the quality of gait post-stroke [76] and bilateral motor coordination [50].

The challenge of bioengineers is to match the most recent neurological findings with the features of the robots developed for gait training post-stroke [3]. These robots should not only simulate physiological patterns but also favour the determinants of a qualitative gait recovery. To stimulate the recovery of a close-to-normal gait movement pattern, the robots should enable variability in lower limb kinematics through sufficient degrees of freedom in all three planes of motion [60]. In addition, they should be flexible and individually adjustable, and they need to encourage active participation from the wearer. The combination of individual support and progression, realtime feedback and guidance, and motor tasks that challenge balance control and coordination, serves for multisensory stimulation that has been suggested to be beneficial for neural reorganization. The optimally developed robot should have the ability to generate a bottom-up and top-down complex and controlled multisensory stimulation aiming to modify the plasticity of neural connections through the experience of moving [5]. 


\begin{tabular}{|c|c|c|c|c|c|c|c|c|c|c|}
\hline \multirow[b]{2}{*}{ Study or Subgroup } & \multicolumn{3}{|c|}{ Intervention } & \multicolumn{3}{|c|}{ Control } & \multirow[b]{2}{*}{ Weight } & Mean Difference & \multirow{2}{*}{$\begin{array}{c}\text { Mean Difference } \\
\text { IV, Random, } 95 \% \mathrm{Cl}\end{array}$} & \multirow{2}{*}{$\begin{array}{l}\text { Risk of Bias } \\
\text { A B C D E }\end{array}$} \\
\hline & Mean & SD & Total & Mean & SD & Total & & IV, Random, $95 \% \mathrm{Cl}$ Year & & \\
\hline Hornby $2008^{\wedge}$ & 0.1 & 0.25 & 24 & 0.3 & 0.74 & 24 & $2.8 \%$ & $-0.20[-0.51,0.11] 2008$ & \begin{tabular}{l|l}
$\longrightarrow$ \\
\end{tabular} & $++0+?$ \\
\hline Hornby $2008^{*}$ & 0.4 & 0.9906 & 24 & 0.4 & 0.9906 & 24 & $0.9 \%$ & $0.00[-0.56,0.56] 2008$ & & $\hookrightarrow+\odot \odot ?$ \\
\hline Westlake 2009 & 0.16 & 0.1914 & 8 & 0.04 & 0.0505 & 8 & $10.8 \%$ & $0.12[-0.02,0.26] 2009$ & - & ๑๑९? \\
\hline Buesing $2015^{\wedge}$ & 0.1 & 0.03 & 25 & 0.1 & 0.04 & 25 & $32.7 \%$ & $0.00[-0.02,0.02] 2015$ & & 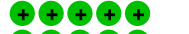 \\
\hline Buesing $2015^{*}$ & 0.01 & 0.04 & 25 & 0.08 & 0.04 & 25 & $32.4 \%$ & $-0.07[-0.09,-0.05] 2015$ & - & $\bullet+$ \\
\hline Tanaka 2019 & 0.06 & 0.1223 & 21 & 0.05 & 0.1362 & 20 & $19.8 \%$ & $0.01[-0.07,0.09] 2019$ & ( & \\
\hline Lee 2019 & 0.6 & 1.0392 & 14 & 0.4 & 0.661 & 12 & $0.7 \%$ & $0.20[-0.46,0.86] 2019$ & & + \\
\hline Total $(95 \% \mathrm{Cl})$ & & & 141 & & & 138 & $100.0 \%$ & $-0.01[-0.07,0.04]$ & 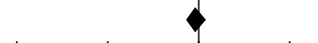 & \\
\hline \multicolumn{5}{|c|}{ Test for overall effect: $Z=0.43(P=0.67)$} & & & & & 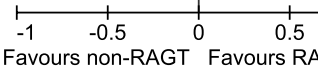 & \\
\hline $\begin{array}{l}\text { Risk of bias legend } \\
\text { (A) Bias arising from } \\
\text { (B) Bias due to devia } \\
\text { (C) Bias due to missi } \\
\text { (D) Bias in measurem } \\
\text { (E) Bias in selection o }\end{array}$ & $\begin{array}{l}\text { ee rando } \\
\text { ons from } \\
\text { goutcom } \\
\text { ent of the } \\
\text { the repc }\end{array}$ & $\begin{array}{l}\text { mization } \\
\text { intended } \\
\text { ne data } \\
\text { outcome } \\
\text { orted resu }\end{array}$ & $\begin{array}{l}\text { proces: } \\
\text { interve } \\
\text { llts }\end{array}$ & $\begin{array}{l}\text { ss } \\
\text { rentions }\end{array}$ & & & & & & \\
\hline \multicolumn{11}{|c|}{$\begin{array}{l}\text { Fig. } 9 \text { A forest plot (generated with the Review Manager Web, The Cochrane Collaboration, 2019, available at revman.cochrane.org) summarizing a } \\
\text { pooled effect estimate on change in spatial symmetry (ratio), following robotic-assisted gait training (RAGT) compared with non-robotic gait training } \\
\text { (non-RAGT). *: assessed during walking at a self-selected velocity SSV; } \wedge \text { : assessed during walking at the fastest velocity possible FV; Cl confidence } \\
\text { interval; } d f \text { degrees of freedom; SD standard deviation }\end{array}$} \\
\hline
\end{tabular}

\section{Conclusion}

This systematic review revealed a substantial knowledge gap underpinning the effects of RAGT post-stroke when compared with non-robotic gait training. Only thirteen eligible randomised controlled trials were identified which evaluated the effects of RAGT post-stroke on objective biomechanical outcome measures. Our findings demonstrated a very low certainty in current evidence for employing RAGT instead of non-robotic gait training to improve gait ability post-stroke. Standardised guidelines for biomechanical quantification of gait should be developed to support researchers in the evaluation of gait-assisting robots used in post-stroke rehabilitation. Well-designed, high-quality clinical trials that complement clinical data with objective, quantitative gait data post-stroke will provide more detailed information on the potential effects of robotic gait training in general, as well as the influence on gait movement pattern in particular. In the long term, this could contribute to the development of RAGT that, either on its own or as an addition to other treatments, can better target true recovery and decrease the impact of compensatory gait patterns post-stroke.

\footnotetext{
Abbreviations

$\mathrm{Cl}$ : Confidence interval; FES: Functional electrical stimulation; FV: Fastest possible velocity; GC: Gait cycle; GRADE: Grading of assessment, development and evaluation; ICF: International classification of functioning, disability and health; MD: Mean difference; O-RAGT: Over ground robotic assisted gait training; PRISMA: Preferred reporting items for systematic review and meta-analysis: RAGT: Robotic assisted gait training; RCT: Randomized clinical trial; SSV: Selfselected velocity; t-RAGT: Treadmill-based robotic assisted gait training; 3D: 3-Dimensional; 2D: 2-Dimensional.
}

\section{Supplementary Information}

The online version contains supplementary material available at https://doi. org/10.1186/s12984-021-00857-9.

Additional file 1: A summary of search terms and results from different databases (Web of Science, Scopus, PubMed, the Cochrane Central Register of Controlled Trials (CENTRAL), Academic Search Premier, Cumulative Index to Nursing and Allied Health Literature [CINAHL], Allied and Complementary Medicine [AMED], ProQuest (Sports Medicine \& Education Index) and Sports Discus)

Additional file 2: A forest plot (generated with the Review Manager Web, The Cochrane Collaboration, 2019, available at revman.cochrane. org) summarizing a pooled effect estimate on change in gait speed $(\mathrm{m} / \mathrm{s})$, following robotic-assisted gait training (RAGT) compared with non-robotic gait training (non-RAGT). Subgroup analyses based on the type of gait robots used: treadmill robotic-assisted gait training (t-RAGT) or overground robotic-assisted gait training (o-RAGT). * : assessed during walking at a self-selected velocity SSV; $\wedge$ : assessed during walking at the fastest velocity possible $\mathrm{FV} ; \mathrm{Cl}$ : confidence interval; $\mathrm{df}$ : degrees of freedom; SD: standard deviation

Additional file 3: A forest plot (generated with the Review Manager Web, The Cochrane Collaboration, 2019, available at revman.cochrane. org) summarizing a pooled effect estimate on change in cadence (steps/ min), following robotic assisted gait training (RAGT) compared with non-robotic gait training (non-RAGT). Subgroup analyses based on the type of gait robots used: treadmill robotic-assisted gait training (t-RAGT) or overground robotic-assisted gait training (o-RAGT). *: assessed during walking at a self-selected velocity SSV; $\wedge$ : assessed during walking at the fastest velocity possible $\mathrm{FV}$; Cl: confidence interval; $\mathrm{df}$ : degrees of freedom; SD: standard deviation

Additional file 4: A forest plot (generated with the Review Manager Web, The Cochrane Collaboration, 2019, available at revman.cochrane.org) summarizing a pooled effect estimate on change in gait speed $(\mathrm{m} / \mathrm{s})$, following robotic assisted gait training (RAGT) compared with non-robotic gait training (non-RAGT). Subgroup analyses based on the year of publication: late studies published 2015-2020 and early studies 2007-2014. *: assessed during walking at a self-selected velocity SSV; $\wedge$ : assessed during walking at the fastest velocity possible $\mathrm{FV}$; $\mathrm{Cl}$ : confidence interval; df: degrees of freedom; SD: standard deviation 
Additional file 5: A forest plot (generated with the Review Manager Web, The Cochrane Collaboration, 2019, available at revman.cochrane. org) summarizing a pooled effect estimate on change in cadence (steps/ min), following robotic assisted gait training (RAGT) compared with nonrobotic gait training (non-RAGT). Subgroup analyses based on the year of publication: late studies published 2015-2020 and early studies 2007-2014. *: assessed during walking at a self-selected velocity SSV; $\wedge$ : assessed during walking at the fastest velocity possible $\mathrm{FV}$; Cl: confidence interval; df: degrees of freedom; SD: standard deviation

\section{Authors' contributions}

$\mathrm{CH}$ conceived the idea. $\mathrm{HN}, \mathrm{AA}, \mathrm{MS}$ and $\mathrm{CH}$ designed the research protocol. $\mathrm{HN}$ did the data search, and HN, AB and MS screened the studies, extracted data and evaluated risk of bias. AA was available to adjudicate any disagreements and reach a consensus. HN performed the quantitative data analysis and drafted the preliminary manuscript. All authors helped in further writing and critical reviewing of the manuscript. $\mathrm{CH}$ obtained the funding. All authors read and approved of the final manuscript.

\section{Funding}

Open access funding provided by Umea University. The research was supported by The Swedish Brain Foundation; Umeå university Foundation for Medical Research; Strategic Funding in Caring Science for Umeå University and Karolinska Institute; The Region Västerbotten County Council; The Foundation for Stroke Research in Norrland, The Nils Erik Holmsten Foundation; and King Gustaf $V$ and Queen Victoria's Foundation of Freemasons. None of the funding bodies had any influence on the design of the study, data collection, analysis, interpretation of data or writing of the manuscript.

\section{Availability of data and materials}

Full search strategy available in Appendix. The datasets analysed during the current study are available from the corresponding author on reasonable request.

\section{Declarations}

Ethics approval and consent to participate Not required.

\section{Consent for publication}

Not applicable.

\section{Competing interests}

All authors declare no competing interests.

\section{Author details}

${ }^{1}$ Department of Community Medicine and Rehabilitation, Physiotherapy, Umeå University, Umeå, Sweden. ${ }^{2}$ Department of Physiotherapy, College of Health Sciences, University of Sharjah, Sharjah, United Arab Emirates.

\section{Received: 23 October 2020 Accepted: 21 March 2021}

\section{Published online: 16 April 2021}

\section{References}

1. Lefeber N, Swinnen E, Kerckhofs E. The immediate effects of robot-assistance on energy consumption and cardiorespiratory load during walking compared to walking without robot-assistance: a systematic review. Disabil Rehabil Assist Technol. 2017;12:657-71.

2. Calabro RS, Cacciola A, Berte F, Manuli A, Leo A, Bramanti A, et al. Robotic gait rehabilitation and substitution devices in neurological disorders: where are we now? Neurol Sci. 2016;37:503-14.

3. Morone G, Paolucci S, Cherubini A, De Angelis D, Venturiero V, Coiro $P$, et al. Robot-assisted gait training for stroke patients: current state of the art and perspectives of robotics. Neuropsychiatr Dis Treat. 2017;13:1303-11.
4. Goffredo M, Guanziroli E, Pournajaf S, Gaffuri M, Gasperini G, Filoni S, et al. Overground wearable powered exoskeleton for gait training in subacute stroke subjects: clinical and gait assessments. Eur J Phys Rehabil Med. 2019;55:710-21.

5. Molteni F, Gasperini G, Cannaviello G, Guanziroli E. Exoskeleton and endeffector robots for upper and lower limbs rehabilitation: narrative review. Pm r. 2018;10:S174-88.

6. Wall A, Borg J, Palmcrantz S. Clinical application of the Hybrid Assistive Limb (HAL) for gait training-a systematic review. Front Syst Neurosci. 2015;9:48.

7. Louie DR, Eng JJ. Powered robotic exoskeletons in post-stroke rehabilitation of gait: a scoping review. J Neuroeng Rehabil. 2016;13:53.

8. Chen G, Chan CK, Guo Z, Yu H. A review of lower extremity assistive robotic exoskeletons in rehabilitation therapy. Crit Rev Biomed Eng. 2013;41:343-63.

9. Pennycott A, Wyss D, Vallery H, Klamroth-Marganska V, Riener R. Towards more effective robotic gait training for stroke rehabilitation: a review. J Neuroeng Rehabil. 2012;9:65.

10. Tedla JS, Dixit S, Gular K, Abohashrh M. Robotic-assisted gait training effect on function and gait speed in subacute and chronic stroke population: a systematic review and meta-analysis of randomized controlled trials. Eur Neurol. 2019;81:1-9.

11. Bruni MF, Melegari C, De Cola MC, Bramanti A, Bramanti P, Calabro RS. What does best evidence tell us about robotic gait rehabilitation in stroke patients: a systematic review and meta-analysis. J Clin Neurosci. 2018;48:11-7.

12. Mehrholz J, Thomas S, Werner C, Kugler J, Pohl M, Elsner B. Electromechanical-assisted training for walking after stroke. Cochrane Database Syst Rev. 2017;5(5):CD006185.

13. Moucheboeuf G, Griffier R, Gasq D, Glize B, Bouyer L, Dehail P, et al. Effects of robotic gait training after stroke: a meta-analysis. Ann Phys Rehabil Med. 2020;63:518-34

14. Tedla Jaya S, Dixit S, Gular K, Abohashrh M. Robotic-assisted gait training effect on function and gait speed in subacute and chronic stroke population: a systematic review and meta-analysis of randomized controlled trials. Eur Neurol. 2019;81:103-11.

15. SchrÖDer J, Truijen S, Van Criekinge T, Saeys W. Feasibility and effectiveness of repetitive gait training early after stroke: a systematic review and meta-analysis. J Rehabil Med (Stiftelsen Rehabiliteringsinformation). 2019:51:78-88.

16. Lo K, Stephenson M, Lockwood C. Effectiveness of robotic assisted rehabilitation for mobility and functional ability in adult stroke patients: a systematic review. JBI Database Syst Rev Implement Rep. 2017;15:3049-91.

17. Bragoni M, Broccoli M, losa M, Morone G, De Angelis D, Venturiero V, et al. Influence of psychologic features on rehabilitation outcomes in patients with subacute stroke trained with robotic-aided walking therapy. Am J Phys Med Rehabil. 2013;92:e16-25.

18. Bernhardt J, Hayward KS, Kwakkel G, Ward NS, Wolf SL, Borschmann K, et al. Agreed definitions and a shared vision for new standards in stroke recovery research: the Stroke Recovery and Rehabilitation Roundtable taskforce. Int J Stroke. 2017;12:444-50.

19. World Health Organization. International classification of functioning, disability and health: ICF. Geneva: World Health Organization; 2017.

20. Geroin C, Mazzoleni S, Smania N, Gandolfi M, Bonaiuti D, Gasperini G, et al. Systematic review of outcome measures of walking training using electromechanical and robotic devices in patients with stroke. J Rehabil Med. 2013;45:987-96.

21. Kwakkel G, Kollen B, Lindeman E. Understanding the pattern of functional recovery after stroke: facts and theories. Restor Neurol Neurosci. 2004;22:281-99.

22. Kautz SA, Duncan PW, Perera S, Neptune RR, Studenski SA. Coordination of hemiparetic locomotion after stroke rehabilitation. Neurorehabil Neural Repair. 2005;19:250-8.

23. Behrman AL, Bowden MG, Nair PM. Neuroplasticity after spinal cord injury and training: an emerging paradigm shift in rehabilitation and walking recovery. Phys Ther. 2006;86:1406-25.

24. Nadeau S, Betschart M, Bethoux F. Gait analysis for poststroke rehabilitation: the relevance of biomechanical analysis and the impact of gait speed. Phys Med Rehabil Clin N Am. 2013;24:265-76. 
25. Moher D, Shamseer L, Clarke M, Ghersi D, Liberati A, Petticrew M, et al. Preferred reporting items for systematic review and meta-analysis protocols (PRISMA-P) 2015 statement. Syst Control Found Appl. 2015;4:1.

26. Liberati A, Altman DG, Tetzlaff J, Mulrow C, Gøtzsche PC, loannidis JP, et al. The PRISMA statement for reporting systematic reviews and meta-analyses of studies that evaluate health care interventions: explanation and elaboration. PLoS Med. 2009;6:e1000100.

27. Richardson WS, Wilson MC, Nishikawa J, Hayward RS. The well-built clinical question: a key to evidence-based decisions. ACP J Club. 1995;123:A12-3.

28. Nuyens GE, De Weerdt WJ, Spaepen Jr AJ, Kiekens C, Feys HM. Reduction of spastic hypertonia during repeated passive knee movements in stroke patients. Arch Phys Med Rehabil. 2002;83:930-5.

29. Schmitt C, Métrailler P. The Motion Maker ${ }^{\mathrm{TM}}$ : a rehabilitation system combining an orthosis with closed-loop electrical muscle stimulation. In: 8th Vienna international workshop on functional electrical stimulation; 2004. p. 117-20.

30. Girone M, Burdea G, Bouzit M, Popescu V, Deutsch JE. A Stewart platformbased system for ankle telerehabilitation. Auton Robots. 2001;10:203-12.

31. Nikitczuk J, Weinberg B, Canavan PK, Mavroidis C. Active knee rehabilitation orthotic device with variable damping characteristics implemented via an electrorheological fluid. IEEE/ASME Trans Mechatron. 2009; 15:952-60.

32. Sterne JAC, Savovic J, Page MJ, Elbers RG, Blencowe NS, Boutron I, et al. RoB 2: a revised tool for assessing risk of bias in randomised trials. BmjBrit Med J. 2019. https://doi.org/10.1136/bmj.14898.

33. Deeks J, Higgins J, Altman D, (editors). Chapter 10. Analysing data and undertaking meta-analyses. In: Higgins J, Thomas J, Chandler J, Cumpston M, Li T, Page M (editors). Cochrane Handbook for Systematic Reviews of Interventions version 60 (updated July 2019). Cochrane 2019. Available at: www.training.cochrane.org/handbook.

34. Geroin C, Picelli A, Munari D, Waldner A, Tomelleri C, Smania N. Combined transcranial direct current stimulation and robot-assisted gait training in patients with chronic stroke: a pilot randomized controlled trial. Physiotherapy (United Kingdom). 2011;97:eS403-4.

35. Brozek JL, Akl EA, Alonso-Coello P, Lang D, Jaeschke R, Williams JW, et al. Grading quality of evidence and strength of recommendations in clinical practice guidelines. Part 1 of 3. An overview of the GRADE approach and grading quality of evidence about interventions. Allergy. 2009:64:669-77.

36. Murad MH, Mustafa RA, Schünemann HJ, Sultan S, Santesso N. Rating the certainty in evidence in the absence of a single estimate of effect. Evid Based Med. 2017;22:85-7.

37. Lewek MD, Cruz TH, Moore JL, Roth HR, Dhaher YY, Hornby TG. Allowing intralimb kinematic variability during locomotor training poststroke improves kinematic consistency: a subgroup analysis from a randomized clinical trial. Phys Ther. 2009;89:829-39.

38. Bang DH, Shin WS. Effects of robot-assisted gait training on spatiotemporal gait parameters and balance in patients with chronic stroke: a randomized controlled pilot trial. NeuroRehabilitation. 2016;38:343-9.

39. Lee HJ, Lee $\mathrm{SH}$, Seo K, Lee M, Chang WH, Choi BO, et al. Training for walking efficiency with a wearable hip-assist robot in patients with stroke: a pilot randomized controlled trial. Stroke. 2019. https://doi.org/ 10.1161/STROKEAHA.119.025950.

40. Westlake KP, Patten C. Pilot study of Lokomat versus manual-assisted treadmill training for locomotor recovery post-stroke. J Neuroeng Rehabil. 2009. https://doi.org/10.1186/1743-0003-6-18.

41. Ogino T, Kanata Y, Uegaki R, Yamaguchi T, Morisaki K, Nakano S, et al. Effects of gait exercise assist robot (GEAR) on subjects with chronic stroke: a randomized controlled pilot trial. J Stroke Cerebrovasc Dis. 2020;29:104886.

42. Srivastava S, Kao PC, Reisman DS, Scholz JP, Agrawal SK, Higginson JS. Robotic assist-as-needed as an alternative to therapist-assisted gait rehabilitation. Int J Phys Med Rehabil. 2016. https://doi.org/10.4172/ 2329-9096.1000370.

43. Husemann B, Muller F, Krewer C, Heller S, Koenig E. Effects of locomotion training with assistance of a robot-driven gait orthosis in hemiparetic patients after stroke: a randomized controlled pilot study. Stroke. 2007:38:349-54.
44. Hidler J, Nichols D, Pelliccio M, Brady K, Campbell DD, Kahn JH, et al. Multicenter randomized clinical trial evaluating the effectiveness of the lokomat in subacute stroke. Neurorehabil Neural Repair. 2009;23:5-13.

45. Tanaka N, Matsushita S, Sonoda Y, Maruta Y, Fujitaka Y, Sato M, et al. Effect of stride management assist gait training for poststroke hemiplegia: a single center, open-label, randomized controlled trial. J Stroke Cerebrovasc Dis. 2019;28:477-86.

46. Buesing C, Fisch G, O'Donnell M, Shahidi I, Thomas L, Mummidisetty CK, et al. Effects of a wearable exoskeleton stride management assist system $\left(S M A^{\circledR}\right)$ on spatiotemporal gait characteristics in individuals after stroke: a randomized controlled trial. J NeuroEng Rehabil (JNER). 2015:12:1-14.

47. Hornby TG, Campbell DD, Kahn JH, Demott T, Moore JL, Roth HR, et al. Enhanced gait-related improvements after therapist-versus roboticassisted locomotor training in subjects with chronic stroke: a randomized controlled study. Stroke. 2008;39:1786-92.

48. Calabrò RS, Naro A, Russo M, Bramanti P, Carioti L, Balletta T, et al. Shaping neuroplasticity by using powered exoskeletons in patients with stroke: a randomized clinical trial. J Neuroeng Rehabil. 2018;15:35.

49. Balaban B, Tok F. Gait disturbances in patients with stroke. PM\&R. 2014;6:635-42.

50. Sharififar S, Vincent HK, Shuster J, Bishop M. Quantifying poststroke gait deviations: a meta-analysis of observational and cross-sectional experimental trials. J Stroke Med. 2019;2:23-31.

51. Chen G, Patten C, Kothari DH, Zajac FE. Gait differences between individuals with post-stroke hemiparesis and non-disabled controls at matched speeds. Gait Posture. 2005;22:51-6.

52. Brandstater ME, de Bruin H, Gowland C, Clark BM. Hemiplegic gait: analysis of temporal variables. Arch Phys Med Rehabil. 1983;64:583-7.

53. Olney SJ, Richards C. Hemiparetic gait following stroke. Part I: characteristics. Gait Posture. 1996:4:136-48.

54. Wonsetler EC, Bowden MG. A systematic review of mechanisms of gait speed change post-stroke. Part 1: spatiotemporal parameters and asymmetry ratios. Top Stroke Rehabil. 2017;24:435-46.

55. Ellis RG, Howard KC, Kram R. The metabolic and mechanical costs of step time asymmetry in walking. Proc Biol Sci. 2013;280:20122784.

56. Jørgensen L, Crabtree NJ, Reeve J, Jacobsen BK. Ambulatory level and asymmetrical weight bearing after stroke affects bone loss in the upper and lower part of the femoral neck differently: bone adaptation after decreased mechanical loading. Bone. 2000;27:701-7.

57. Hsu A-L, Tang P-F, Jan M-H. Analysis of impairments influencing gait velocity and asymmetry of hemiplegic patients after mild to moderate stroke11 No commercial party having a direct financial interest in the results of the research supporting this article has or will confer a benefit upon the authors(s) or upon any organization with which the author(s) is/are associated. Arch Phys Med Rehabil. 2003;84:1185-93.

58. Wang Y, Mukaino M, Ohtsuka K, Otaka Y, Tanikawa H, Matsuda F, et al. Gait characteristics of post-stroke hemiparetic patients with different walking speeds. Int J Rehabil Res. 2020;43:69-75.

59. Allen JL, Kautz SA, Neptune RR. Step length asymmetry is representative of compensatory mechanisms used in post-stroke hemiparetic walking. Gait Posture. 2011;33:538-43.

60. Pennycott A, Wyss D, Vallery H, Klamroth-Marganska V, Riener R. Towards more effective robotic gait training for stroke rehabilitation: a review. J NeuroEng Rehabil (JNER). 2012;9:65

61. Cho JE, Yoo JS, Kim KE, Cho ST, Jang WS, Cho KH, et al. Systematic review of appropriate robotic intervention for gait function in subacute stroke patients. Biomed Res Int. 2018;2018:4085298.

62. Moseley A, Wales A, Herbert R, Schurr K, Moore S. Observation and analysis of hemiplegic gait: stance phase. Aust J Physiother. 1993;39:259-67.

63. Bensoussan L, Mesure S, Viton JM, Delarque A. Kinematic and kinetic asymmetries in hemiplegic patients' gait initiation patterns. J Rehabil Med. 2006;38:287-94

64. Woolley SM. Characteristics of gait in hemiplegia. Top Stroke Rehabil. 2001;7:1-18.

65. Hornby TG, Straube DS, Kinnaird CR, Holleran CL, Echauz AJ, Rodriguez KS, et al. Importance of specificity, amount, and intensity of locomotor training to improve ambulatory function in patients poststroke. Top Stroke Rehabil. 2011;18:293-307.

66. Sarasola-Sanz A, Irastorza-Landa N, Lopez-Larraz E, Bibian C, Helmhold F, Broetz D, et al. A hybrid brain-machine interface based on EEG and 
EMG activity for the motor rehabilitation of stroke patients. In: IEEE International Conference on Rehabilitation Robotics: [proceedings]. 2017:2017:895-900.

67. Li M, Xu G, Xie J, Chen C. A review: motor rehabilitation after stroke with control based on human intent. Proc Inst Mech Eng H. 2018;232:344-60

68. Lennon O, Tonellato M, Del Felice A, Di Marco R, Fingleton C, Korik A, et al. A systematic review establishing the current state-of-the-art, the limitations, and the DESIRED checklist in studies of direct neural interfacing with robotic gait devices in stroke rehabilitation. Front Neurosci. 2020;14:578.

69. Jones TA, Adkins DL. Motor system reorganization after stroke: stimulating and training toward perfection. Physiology (Bethesda). 2015;30:358-70.

70. French B, Thomas LH, Coupe J, McMahon NE, Connell L, Harrison J, et al. Repetitive task training for improving functional ability after stroke. Cochrane Database Syst Rev. 2016;11:Cd006073.

71. Van Peppen RP, Kwakkel G, Wood-Dauphinee S, Hendriks HJ, Van der Wees PJ, Dekker J. The impact of physical therapy on functional outcomes after stroke: what's the evidence? Clin Rehabil. 2004;18:833-62.
72. Whitall J. Stroke rehabilitation research: time to answer more specific questions? Neurorehabil Neural Repair. 2004;18:3-8; author reply 9-11.

73. Krasovsky T, Levin MF. Review: toward a better understanding of coordination in healthy and poststroke gait. Neurorehabil Neural Repair 2010;24:213-24.

74. Wonsetler EC, Bowden MG. A systematic review of mechanisms of gait speed change post-stroke. Part 2: exercise capacity, muscle activation, kinetics, and kinematics. Top Stroke Rehabil. 2017;24:394-403.

75. Van Criekinge T, Saeys W, Hallemans A, Velghe S, Viskens PJ, Vereeck L, et al. Trunk biomechanics during hemiplegic gait after stroke: a systematic review. Gait Posture. 2017;54:133-43.

76. Kwakkel G, Lannin NA, Borschmann K, English C, Ali M, Churilov L, et al. Standardized measurement of sensorimotor recovery in stroke trials: consensus-based core recommendations from the stroke recovery and rehabilitation roundtable. Neurorehabil Neural Repair. 2017;31:784-92.

\section{Publisher's Note}

Springer Nature remains neutral with regard to jurisdictional claims in published maps and institutional affiliations.
Ready to submit your research? Choose BMC and benefit from:

- fast, convenient online submission

- thorough peer review by experienced researchers in your field

- rapid publication on acceptance

- support for research data, including large and complex data types

- gold Open Access which fosters wider collaboration and increased citations

- maximum visibility for your research: over 100M website views per year

At BMC, research is always in progress.

Learn more biomedcentral.com/submissions 